\title{
Bus control strategies in corridors with signalized intersections
}

\author{
Miquel Estrada $^{* a}$, Josep Mensión $^{* b}$, Josep M. Aymami*c and Laura Torres*d \\ ${ }^{*}$ a Associate Professor. Center for Innovation in Transport (CENIT) . Universitat Politècnica de Catalunya- BarcelonaTECH, \\ C. Jordi Girona 1-3, B1-113 08034 Barcelona, Spain. miquel.estrada@upc.edu. Corresponding author. \\ ${ }^{* b}$ Director of Central Services and Deputy Chief Officer of Bus Network. Transports Metropolitans de Barcelona (TMB). \\ C. 60, n. ${ }^{\circ}$ 21-23, sector A, Pol. Ind. de la Zona Franca, 08040, Barcelona, Spain. jmension@tmb.cat \\ ${ }^{{ }^{*} \mathrm{c}}$ Regional manager. Business Development. Transport Simulation Systems (TSS). C. Ronda Universitat 22B, 08008 \\ Barcelona, Spain. josep.aymami@aimsun.com \\ ${ }^{*}$ C Consultant engineer. Transport Simulation Systems (TSS). C. Ronda Universitat 22B, 08008 Barcelona, Spain. \\ laura.torres@aimsun.com
}

This paper proposes a new dynamic bus control strategy aimed at reducing the negative effects of timeheadway variations on route performance, based on real-time bus tracking data at stops. In routes with high demand, any delay of a single vehicle ends up causing an unstable motion of buses and producing the bus bunching phenomena. This strategy controls the cruising speed of buses and considers the extension of the green phase of traffic lights at intersections, when a bus is significantly delayed. The performance of this strategy will be compared to the current static operation technique based on the provision of slack times at holding points. An operational model is presented in order to estimate the effects of each controlling strategy, taking into account the vehicle capacity constraint. Control strategies are assessed in terms of passenger total travel time, operating cost as well as on the coefficient of headway variation. The effects of controlling strategies are tested in an idealized bus route under different operational settings and in the bus route of highest demand in Barcelona by simulation. The results show that the proposed dynamic controlling strategy reduces total system cost (user and agency) by $15-40 \%$ as well as the coefficient of headway variation $53-78 \%$ regarding the uncontrolled case, providing a bus performance similar to the expected when time disturbance is not presented.

Keywords: Bus bunching; dynamic controlling strategies; time-headway adherence, bus reliability 


\section{INTRODUCTION}

The reliability of transit modes is an important issue to ensure their competitiveness against the extended use of private cars in major cities. However, in overall surface transit services with partial right of way, route travel times are highly dependent to transit demand and traffic states. There are several reasons in these systems that cause service disruptions such as illegal freight loading/unloading operations, taxi stops, use of bus lanes by slow vehicles (bikes, street sweepers) or car merging operations due to right turns. These facts, combined with transit demand fluctuations at stops and traffic light settings, make it difficult to maintain time-headway adherence and control the transit system performance. In bus routes with high demand, when a single bus is delayed from its schedule, the number of waiting passengers will increase at the following stops, resulting in a higher vehicle delay. This local disruption propagates to the whole fleet producing vehicle bunching, irregular vehicle arrivals at stops, unstable time-headways and higher user waiting times.

Some research has been done to describe the dynamic performance of the bus system operations. Newell and Potts (1964) and Osuna and Newell (1972) were the first contributions that described the unstable performance of a cyclic bus fleet operation. In order to tackle the bus bunching problem, several control strategies are available. Traditionally, the bus pairing has been mitigated allocating slack times in bus schedules at determined stops (holding points) along the route (Barnett, 1974; Turnquist, 1981; and Rossetti and Turitto, 1998). Slack times should compensate the delays of those buses experiencing random disruptions so that the schedule adherence would be still satisfied. Nevertheless, the obligation that all buses must remain a common slack time in a holding point represents a reduction of commercial speed. Indeed, it causes a significant inefficiency in the system's productivity. Moreover, this control strategy for maintaining the schedule of a single bus, does not take into account the real performance of the others. Therefore, some studies propose dynamic control strategies to monitor the response of the whole fleet to random disruptions in a short time horizon (Eberlein et al. 2001; Dessouky et al. 2003; Adamski and Turnau, 1998). These contributions determine the location of a holding point and a specific amount of slack time for each bus, based on suboptimal procedures and the dynamic bus performance data. Real-time information is supposed to be available, as Automated Vehicle Location (AVL) and Automatic Passenger Counter (APC) systems will be equipped in the vehicles. In $\mathrm{Yu}$ and Yang (2007) an improved holding-point optimization procedure is presented based on genetic algorithms to minimize total passenger costs. Other contributions develop optimization models based on holding points and stop skipping strategy, where the performance of the bus system is predicted over a rolling horizon. This prediction is made considering that all variables are deterministic and known in advance (Delgado et al. 2012) or even stochastic (Sáez et al., 2012; Cortés et al., 2010). Fonzone et al. (2015) proposed bus overtaking at stops in order to accommodate better the waiting passengers in buses that didn't reach its vehicle capacity constraint.

Although the previous contributions are generally based on short term predictions of the system behavior, other approaches propose adaptive strategies to the real performance of buses. They actuate over the system variables in the interstation segments of a single bus route. Based on control theory principles, Daganzo (2009) defines an adaptive variable cruising speed patterns for public transportation vehicles. This control strategy may be conceived as dynamic holding times in a segment of the route: if a fast vehicle is catching up the vehicle ahead, the speed of the former vehicle is linearly reduced with regard to the difference between the target and the actual headway. The results provided by this method outperform the former static holding point strategies in terms of system productivity and regularity. Nevertheless, this procedure does not respond properly when the headway adherence is significantly poor. Daganzo and Pilachowski (2011) improved the determination of the cruising speed pattern when the time-headway variance is significant. In Xuan et al. (2011), a family of dynamic holding strategies are presented to improve both user and operating costs. This method improves the efficiency of existing control strategies since it minimizes the required slack times by $40 \%$ compared to conventional schedule-based methods. In Bartholdi and Eisenstein (2012), a method based on Markov-chains is presented where headways are dynamically self-equalized to a natural value. In addition to that, Argote-Cabanero et al. (2015) extends a dynamic control method for several interacting bus routes. The proposed method consists of a combination of dynamic holding and driver guidance that shows the proper cruising speed of buses along the route based on real-time data.

As is stated in Muñoz et al. (2015), previous contributions based on control theory assume that buses have infinite capacity to accommodate all the passengers waiting at stops. However, the scalable reduction of bus speeds in high transit demand corridors may lead to a problem of vehicle capacity. Experience shows that some users cannot get on overcrowded buses arriving at the stop and need to wait to the following transit vehicles. Indeed, both holding point and dynamic speed strategies are aimed to guarantee the time-headway adherence at the expense of losing commercial speed (in the whole fleet or passenger travel time) and increasing 
operating costs. Nevertheless, few contributions assessed the cost in which transit agencies will incur to deal with bus bunching. Indeed, transit agencies would take advantage of dynamic transit signal priority measures in order to minimize the reduction of the vehicle speed due to the time spent at holding points. In TRB (2013) there is an extended analysis of different techniques of transit vehicle actuated strategies that design off-line and online synchronization of traffic signals. The connection of buses to the transit control center (TCC) and the deployment of a coordinated Transit System Priority (TSP) system may significantly reduce the bus delay by $55-75 \%$ with regard to static transit priority systems (Hu et al. 2015).

To our knowledge, there are no contributions analyzing how traffic signal priority may help the system to maintain a good regularity. Therefore, this paper proposes an adaptive dynamic bus control strategy, based on active signal priority for buses. Taking into account real-time headway information and traffic signal variables, we propose an adaptive transit speed pattern combined with a signal offset modification at specific intersections, to avoid the bus bunching effect. The adaptive transit speed pattern has been adapted from the contributions of Daganzo (2009) and Daganzo and Pilachowski (2011). All stops are conceived as check points where the timeheadway adherence control is estimated using AVL technologies. When the time-headway of one bus (with respect to the bus ahead) is larger than a targeted value, the green phase of downstream traffic lights may be extended (constrained to a maximum value) to allow the bus to pass through the signal without stopping. At the same time, the speed of buses showing smaller time-headways with regard to the target value with the vehicle ahead, will be reduced. However, in this paper, this speed reduction is lower than the presented in Daganzo and Pilachowski (2011). This strategy outperforms comparatively user costs and the coefficient of headway variation with regard to existing control procedures. Besides, it also improves the operating costs, since no additional vehicles are required in comparison to slack time strategies. Moreover, the modeling approach alleviates some of the drawbacks of the former contributions as stated in Muñoz et al. (2015): the occupancy of the vehicles is considered when activating the control criteria. However, it requires that APC systems should be deployed in vehicles to put in practice these control strategies.

Although the largest bus transit agencies in developed countries have already deployed expensive AVL systems, in the recent years, affordable technology has arisen to trace each bus in the line. Last developments use simple smart phones equipped in each bus with a single ad-hoc application to implement coordinated dynamic speed control strategy in a bus corridor. On the other hand, the development of Radio Frequency Identification Devices (RFID) of large range, allows the communication between vehicles with the infrastructure. This cheap technology is currently able to recognize a specific bus at upstream sections of traffic light intersections and activate some modifications of signal settings (TSP). The integration of the former technologies would make it possible for any kind of transit agency all over the world to deal with the schedule adherence problem.

Moreover, the time-headway adherence problem will be a crucial issue for those bus agencies that are willing to deploy full electric vehicles in routes to mitigate the local emissions and Green House Gases (GHG). In fact, European Union is fostering electromobility services in cities by means of several research projects (ZeEUS and Eliptic). Different bus technologies and charging infrastructure solutions will be analyzed in demonstration sites. Based on the experience gained by the authors in these projects, it can be stated that there is not any fully- electric bus of 18 meters of length in the market (or even an articulated bus prototype) able to provide continuous service (15-16 hours per day) with an initial charge at the bus garage (September 2015). All of them need on-route charging operations at charging stations located along the route. The slack time reserved at specific holding stops should be sufficient to perform these charging operations under perfect time-headway adherence conditions. Nevertheless, if the bus arrivals at these points are irregular, vehicles cannot be charged at full capacity, unless some queues of vehicles at the charging stations appear (causing more disturbances and schedule variance) or redundant charging stations (more servers) are deployed.

\section{MODELING FRAMEWORK}

A dynamic bus following model similar to that introduced in Daganzo (2009) is presented for describing the physics of bus behavior and their trajectories. This method may be adaptive to the actual performance of the bus system. It can reproduce strategies controlling the headway variation and oscillatory effects.

We consider a straight bus route of length $2 L$ as it is depicted in Figure 1. Buses run along the route in two directions (from A to B and from B to A). The route presents $2 N$ bus stops, where the distance between stop $s$ and $s+1$ is denoted by $l_{s}$. Let $J$ be the total number of buses operating the route in the two directions. Each bus is labeled by $j=1, \ldots, J$ and is supposed to travel the roundtrip, stopping at each stop $s, s=1, . .2 N$. It is considered that bus $j=j^{*+1}$ is in the rear of bus $j^{*}\left(j^{*}=1, \ldots, J-1\right)$. Since buses may operate the route several cycles, the stops are labeled by $s=1+(k 2 N), 2+(k 2 N), . ., 2 N+(k 2 N)$, where $k(0 \leq k<\infty)$ is an integer number that denotes the completed number of round trips made by the bus in the line. Stops $s=1+(k 2 N)$ and $s=N+1+(k 2 N)$ denote the 
starting points for each route direction trip. Thus, stop pairs $(s=1+k 2 N ; s=2 N+k 2 N)$ and $(s=N+k 2 N$; $s=N+1+k 2 N$ ) represent the same physical point (terminals or headers) but refers to bus stops belonging to different directions of service.

We also assume that buses run along a corridor equipped with a set of $I$ signalized intersections controlled by the Traffic Control Center. Each intersection $i=1,2, . ., I$ is characterized by the traffic signal cycle time $\left(C_{i}\right)$ and the green offset with regard to a general reference clock $\Delta_{i}\left(0 \leq \Delta_{i}<C_{i}\right)$. For the sake of simplicity, we consider that the signal cycle time consists of a green phase time $\left(g_{i}\right)$ followed by the red phase time $\left(r_{i}\right)$.

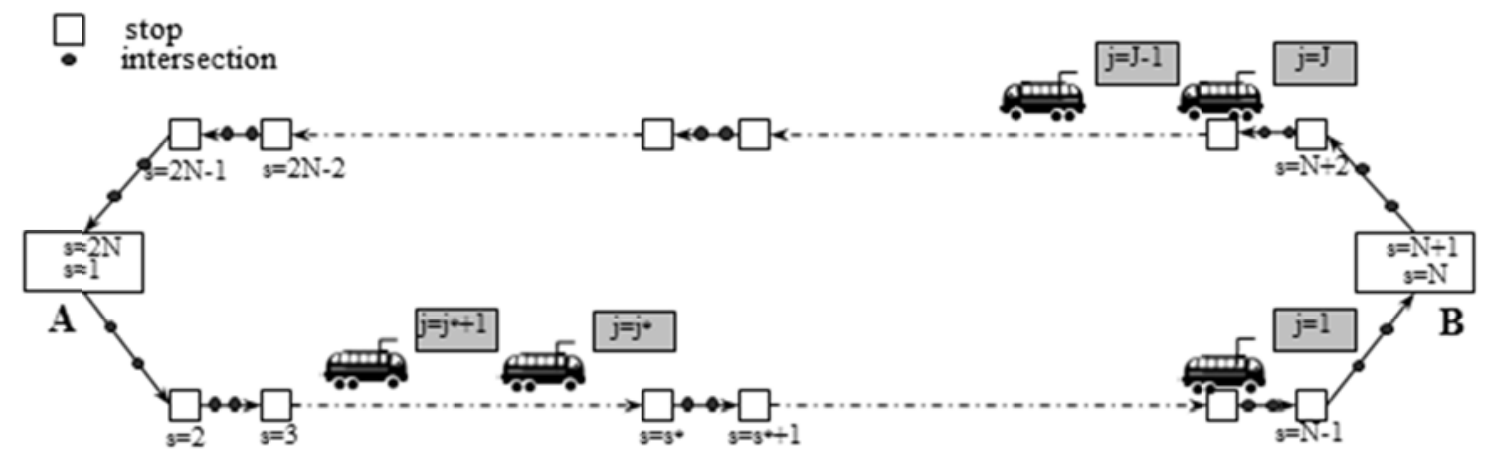

\section{FIGURE 1 Schematic illustration of the bus route}

Equation (1) defines the number of vehicles needed $(J)$ in the route to maintain the targeted bus headway $H$ as a function of the bus travel time in a roundtrip. It is estimated as the sum of four time components for each route segment between stop $s$ and $s+1$ : running time, $\left(T_{r, s}\right)$, time spent at intersections $\left(T_{p, s}\right)$, time spent at stop $s$ $\left(T_{\mathrm{s}}\right)$ and the slack time introduced in schedule at stop $s$ in order to compensate potential service disruptions $\left(\phi_{s}\right)$. The fourth term allows us the assessment of "static holding point strategies" to tackle bus bunching. In addition to that, it is supposed that at terminals $\mathrm{A}$ and $\mathrm{B}(s=N+k 2 N$ and $s=2 N+k 2 N)$ layover times $\theta_{A}$ and $\theta_{B}$ are allocated to let drivers rest an amount of time before continuing the service. This extra time is defined by a mandatory labor rule for each agency and it is independent of the slack time devoted at holding points. The mathematical operator $[x]^{+}$denotes the upper integer of the term $x$.

$$
J=\left[\frac{\sum_{s=1}^{2 N}\left[T_{r, s}+T_{p, s}+T_{s}+\phi_{s}\right]+\theta_{A}+\theta_{B}}{H}\right]^{+}
$$

The travel time of bus $j$ between stops $s$ and $s+1\left(T_{j}(s)\right)$ can be calculated by Equation $(2)$, where $v_{j}(s)$ is the cruising speed of bus $j$ in this section. If the time headway is perfectly regular, the bus cruising speed is supposed to be the maximal, $v_{b}$. We will see later that one of the strategies to tackle bus bunching will consist on the modification of the speed of bus $j$ ).

$$
T_{j}(s)=T_{r, s}+T_{p, s}=\frac{l_{s}}{v_{j}(s)}+\sum_{p=1}^{I_{s}} d_{j, p}
$$

The first term of Equation (2) captures the running time of bus $j$ in the segment between stops ( $s$; $s+1)$. The second term represents the delay caused by traffic signals on the bus performance. We suppose that the section between stops $(s ; s+1)$ encompasses a total amount of $I_{s}$ intersections $\left(I_{s}<|I|\right)$. The variable $d_{j, p}$ is the time that bus $j$ waits at each intersection $p \in I_{s}$ in the route section between stops $(s, s+1)$. If bus $j$ arrives at intersection $p$ when the green phase is active, the variable $d_{j, p}$ will be equal to 0 . In other situations, the bus 
trajectory needs to be modified.

Equation (3) allows the evaluation of the arrival time at intersection $p, t_{j, p}^{a}$, based on the departure time at the previous intersection or stop $\left(t_{j, p-1}^{d}\right)$ and the location of intersections $(p-1, p)$, as it is depicted in Figure 2 . It is supposed that the length $x_{p}$ between the location of intersection $p$ with regard to the first stop is known. Equation (4) establishes the number of signal cycles of $C_{p}$ time $\left(n_{j, p}{ }^{*}\right)$ that have been completed before the arrival of bus $j$ at intersection $p$, where $[x]^{-}$denotes the mathematical operator estimating the lower integer of $x$. From this value, it is possible to determine the departure time at intersection $p$ as well as the total signal delay time by Equation (5) and (6) respectively. The first case of Equation (5) determines that bus $j$ arrives at intersection $p$ when the green phase is activated; consequently, there is no vehicle delay. Otherwise, the second case represents that the traffic signal is red when this bus arrives at this intersection. Therefore, its departure must be postponed to the green phase of the next signal cycle $C_{p}$. Finally, the delay at intersection $p$ is assessed in Equation (6) as the difference between the departure and arrival time of bus $j$ at this intersection. The time spent in accelerating/ deaccelerating the vehicles up to/from the cruising speed due to a stop or a traffic light is neglected.

$$
\begin{aligned}
& t_{j, p}^{a}=t_{j, p-1}^{d}+\left[\frac{x_{p}-x_{p-1}}{v_{j}(s)}\right] \quad p=2, . ., I_{s} \\
& n_{j, p}^{*}=\left[\frac{t_{j, p}{ }^{a}-\Delta_{p}}{C_{p}}\right]^{-} \\
& t_{j, p}{ }^{d}= \begin{cases}t_{j, p}{ }^{a} & \text { if } t_{j, p}{ }^{a} \leq\left(C_{p} n_{j, p} *+\Delta_{p}+g_{p}\right) \\
C_{p}\left(n_{j, p}^{*}+1\right)+\Delta_{p} & \text { in other cases }\end{cases} \\
& d_{j, p}=t_{j, p}{ }^{d}-t_{j, p}{ }^{a}
\end{aligned}
$$

The estimation of the arrival time of bus $j$ to the first intersection of the section $(s ; s+1)$, i.e. $p=1$, is made by Equation (3). In this Equation, the term $t_{j, p-1}^{d}$ should be replaced by the departure time of the last stop $s$ $\left(t_{j}^{d}(s)\right)$ and $x_{p-1}$ by the coordinate of stop $s\left(x_{s}\right)$.

Furthermore, the time that each bus $j$ spent at each stop $s$ is evaluated as a function of the number of boarding and alighting passengers. We assume that $Y_{o d}^{t}$ is the O-D matrix which defines the passenger flow at time interval $t$ that boards at stop $o$ and alights at stop $d(o=1, . ., N-1 ; d=o+1, . . N$ in direction A-B; $o=N, . .2 N-1$; $d=o+1, . ., 2 N$ for direction B-A). The total passenger flow in one direction of service can be calculated as $q=\sum_{o=1}^{2 N-1} \sum_{d>o}^{2 N} Y_{o d}^{t}$. Therefore, the percentage of passengers travelling between stops $(o, d)$ in time interval $t$ is evaluated by $y_{o d}^{t}=(1 / q) Y_{o d}^{t}$. In Section 3 , the performance of the bus route will be assessed, keeping the percentage of the passenger flow distribution constant between stops $\left(y_{o d}^{t}\right)$, and scaling the total passenger demand $q$ in the route.

The time interval $t$ may have different time lengths, from minutes to several hours. It depends on how the information has been obtained from the real world (on-board O-D survey, boarding alighting counters). Although real implementations usually have estimations for the passenger O-D matrix, aggregated in hours or even for the whole day, the variation of $Y_{o d}^{t}$ over short domains of time intervals implies a significant disturbance of the dwell time at stops and consequently of the headway adherence. Hence, the number of passengers alighting $\left(a_{j}(s)\right)$ and boarding $\left(b_{j}(s)\right)$ at stop $s(s=1, \ldots, N-1)$ for each bus $j$ when the headway adherence is perfect, may be estimated using Equation (7a) and (7b). The term $(H q)$ captures the total number passengers that have got on bus $j$ in the whole direction of service (A-B). We sum the passenger flow percentages from all potential origins $(k=1, \ldots s-1)$ to the stop $s$, when the alighting passengers of bus $j$ at stop $s$ is addressed in Equation (7a). The boarding passengers are addressed in a similar way, adding the flow percentage from stop $s$ to all potential downstream destinations $(k=S+1, . ., N)$. The term $g_{j k}$ is equal to 1 if the arrival of vehicle $j$ at stop $k$ is made in the time interval $t^{*}\left(1 \leq t^{*} \leq F\right)$ and 0 otherwise. The parameter $F$ is the number of subsets of stationary time periods in which the passenger flow distribution among stops is evaluated. To be consistent, the boarding and alighting demand values at starting and ending stops of a route must defined $\left(a_{1}=a_{N+1}=b_{N}=b_{2 N}=0\right)$. The calculation in direction B-A can be done easily adapting Equations (7) to the corresponding passenger flows and demand patterns. If we do not find empirical data to estimate boarding and alighting passengers, Equations (7a) and (7b) may be substituted by stochastic functions according to the assumption of probabilistic distributions of 
these variables.

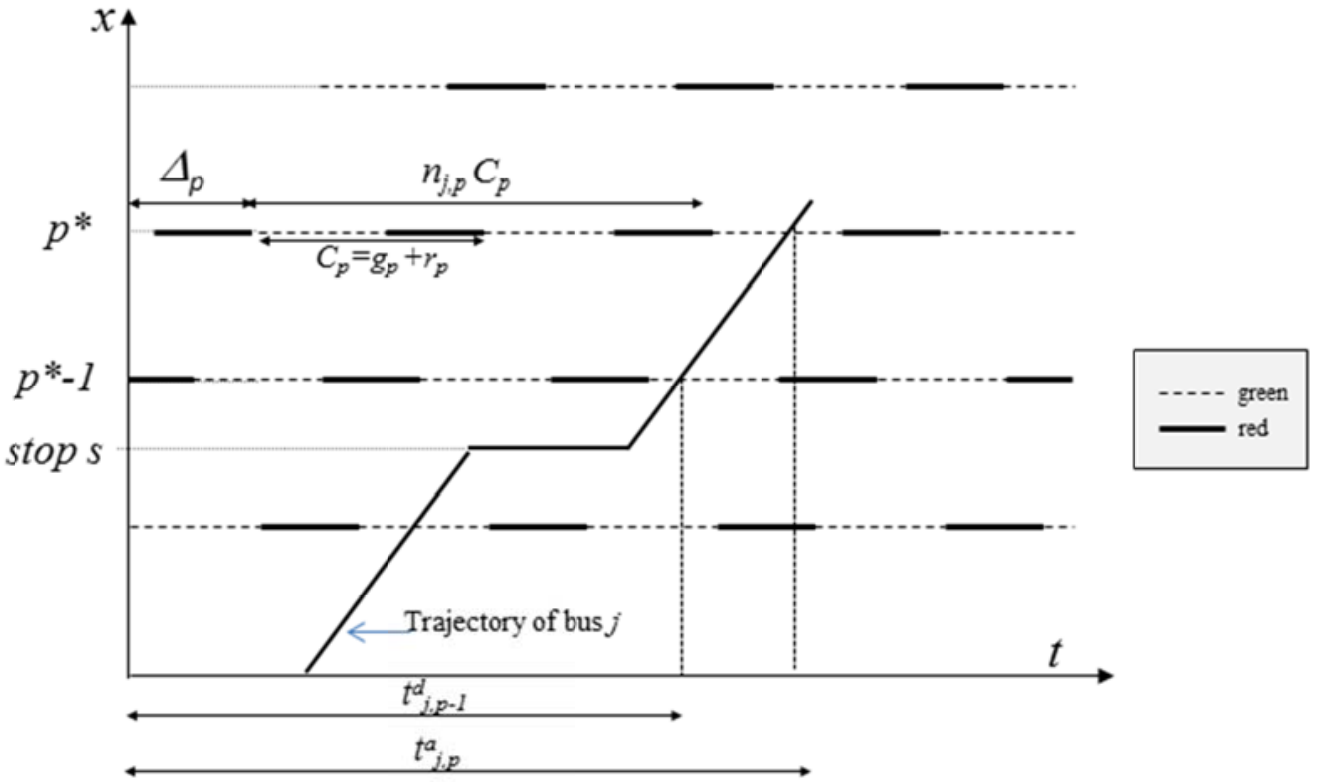

FIGURE 2 Schematic representation of the trajectory of bus $j$

$$
\begin{aligned}
& a_{j}(s)=H q \sum_{k=1}^{s-1} \sum_{t=1}^{F} y_{k s}^{\mathrm{t}} g_{j k} \quad \text { in direction A - B } \\
& b_{j}(s)=H q \sum_{k=s+1}^{N} \sum_{t=1}^{F} y_{s k}^{\mathrm{t}} g_{j k} \quad \text { in direction A - B }
\end{aligned}
$$

Thus, the time spent by each bus at stop $s$ when system is totally regular $\left(T_{s}\right)$ can be calculated by Equation (8)- The parameter $t_{o c}$ is a constant time devoted to the door opening and closing operations of each vehicle and parameters $\gamma, \eta$ are respectively the unit boarding and alighting time per passenger. Equation (8) states that the boarding and alighting operations are performed independently by differentiated doors.

$$
T_{s}=t_{o c}+\max \left[\not b_{s} ; \eta a_{s}\right]
$$

Once the bus travel time has been estimated for each phase of the route roundtrip (links, intersections, stops and holding points) in a regular state, the bus motion model is described by Equations (9) - (11). The variables $t_{j}^{d}(s-1), t_{j}^{a}(s)$ refer to the departure time of bus $j$ from stop $(s-1)$ and its arrival time at stop $s$ respectively. The variable $t_{j}^{d}(s-1)$ is estimated through Equation (9) as a function of the arrival time of the bus of study $j$ at the previous stop $(s-1)$ and the corresponding dwell time at this stop $s-1$. The variables $B_{j}(s-1)$ and $A_{j}(s-1)$ determine the total number of boarding passengers and alighting passengers at stop $(s-1)$ respectively. When the system is totally regular (perfect time-headway adherence), we assume that $B_{j}(s-1)=b_{j}(s-1)$ and $A_{j}(s-$ $1)=a_{\mathrm{j}}(s-1)$ respectively.

However, the calculation of arrival times at stops needs to identify three different cases of studies. When the stop under analysis is not one of the first stops of the two route directions (terminals), arrivals are estimated with regard to the travel time from the previous stop and the potential delays at intersections (first case of Equation 10). Nevertheless, when we analyze the variable $t_{j}^{a}(s)$ at terminals $(s=1+2 k N$ or $s=N+1+2 k N)$, we should take into account the layover and slack times. If the arrival of bus $j$ and its passenger alighting process at the ending stop is made without a relevant delay, this bus $j$ may start running in the opposite direction trip, satisfying a perfect correspondence to the target headway. It means that buses should depart at each $H$ units of time. Therefore, we add $k J$ times the headway $H$ to the arrival time at this stop of each bus $j$ in the first round cycle, where the time disturbance is still not present in the system. This situation is represented in the third case of Equation (10). Finally, if that bus has an important arrival delay at the last stop of one direction, there wouldn't be enough slack time in the terminal to compensate this irregularity. Therefore, bus $j$ would not start 
from the first stop at the target time headway $H$ (second case of Equation 10) so that the disturbances would still be propagating in the opposite direction trip. In that case, the vehicle starts the service in the opposite direction just after being held the mandatory lay-over time $\left(\theta_{\min }\right)$ at this terminal.

The model only needs the insertion time of each vehicle $j(j=1, . ., J)$ in the system to properly characterize its trajectory along the route. This information can be defined by Equation (11).

$$
\begin{aligned}
& t_{j}^{d}(s-1)=t_{j}^{a}(s-1)+t_{o c}+\max \left(\gamma B_{j}(s-1) ; \eta A_{j}(s-1)\right) \\
& t_{j}^{a}(s)= \begin{cases}t_{j}{ }^{d}(s-1)+\frac{L}{v_{j}(s-1)}+\sum_{p} t_{p}^{r} \quad \text { if } s \neq 1+(2 k N) \text { and } s \neq N+1+(2 k N) \\
t_{j}{ }^{d}(s-1)+\theta_{\text {min }} & \text { if } t_{j}^{d}(s-1)+\theta_{\text {min }}>t_{j-1}{ }^{a}(s)+H \text { and } s=1+(2 k N) ; N+1+(2 k N) \\
t_{j}{ }^{a}(s-2 k N)+k J H & \text { if } t_{j}{ }^{d}(s-1)+\theta_{\text {min }} \leq t_{j-1}{ }^{a}(s)+H \text { and } s=1+(2 k N) ; N+1+(2 k N)\end{cases} \\
& t_{j}^{a}(1)=(j-1) H \quad j=1, \ldots, J
\end{aligned}
$$

\subsection{Modelling the unstable motion of buses}

The analysis of bus system performance under service disruptions is conducted by the insertion of an extra time $U_{j}(s)$ in the arrival time of specific bus $j$ to stop $s$ (Equation 10). The exogenous variable $U_{j}(s)$ represents the potential delay that bus $j$ may experience during the trip between stops $(s-1 ; s)$. It would cause the headway variation among the whole fleet. Moreover, the model takes into account the traffic signal settings along the corridor. When the time-headway of the bus route is not multiple of the signal cycle time $\left(H / C_{p}\right.$ is not an integer number), buses will find a different sequence of green-red phases at intersections. This is an additional source of instability in the bus performance. The proposed dynamic model will analyze the performance of the system and passengers behavior because of this alteration $U_{j}(s)$, considering the current signal settings in the route.

In this state of service irregularity, the assumption regarding the estimation of terms $B_{\mathrm{j}}(s-1)=b_{j}(\mathrm{~s}-1)$ and $A_{\mathrm{j}}(s-1)=a_{\mathrm{j}}(\mathrm{s}-1)$ is not valid. The number of boarding passengers of vehicle $j$ at stop $(s-1)$ will directly depend on the real headway with the bus operating ahead. As we track the arrival and departure time of all vehicles at the overall bus stops, the evaluation of terms $B_{\mathrm{j}}(s-1)$ and $A_{\mathrm{j}}(s-1)$ can be easily done by Equations (12)-(13). The term $\left(t_{j}{ }^{a}(s-1)-t_{j-1}{ }^{a}(s-1)\right)$ represents the current time-headway between buses $(j, j-1)$. On one hand, the boarding passengers on bus $j$ at stop $s$ will depend on the waiting passengers at this stop, term $D_{j-1}(s-1)$ in Equation

(12). The model here also improves the existing contributions in bus bunching because it takes into account the vehicle capacity constraint. This constraint is addressed in the following Equations (14)-(15). Therefore, the total number of passengers that cannot get on the previous bus $(j-1), D_{j-1}(s-1)$, also contributes to the number of boarding passengers on bus $j$ at stop $s-1$, calculated in Equation (12). The summation of Equation (12) represents the number of trips carried out, in the same direction of service, between stop $(s-1)$ and all potential downstream destinations $(m, m>s-1)$. Therefore, parameter $k^{*}$ denotes the number of roundtrips completed from the initial time of study. Parameter $\beta$ refers to the route direction in the roundtrip where bus $j$ is running ( $\beta=0$ for direction $\mathrm{A}-\mathrm{B}$ and $\beta=1$ for direction $\mathrm{B}-\mathrm{A})$.

On the other hand, the number of alighting passengers at stop $s-1, A_{j}(s-1)$, does not depend on the headway between buses but, on the current onboard passengers of bus $j$ alighting at this stop. In equation (13), we assume that the number of alighting passengers at stop $s$ is proportional to the ratio of the hourly passenger flow to stop destination $s$ divided by the total demand of the static hourly O-D matrix.

$$
\begin{aligned}
& B_{j}(s-1)=\left(t_{j}{ }^{a}(s-1)-t_{j-1}{ }^{a}(s-1)\right) \sum_{m>s-1}^{\left(2+2 k^{*}-\beta\right) N} q y_{s-1 m}^{t}+D_{j-1}(s-1) \quad s>1 \\
& \text { where } \quad k * 1+2 k * N \leq s \leq 2 N+2 k * N ; k^{*} \in \aleph \\
& \beta= \begin{cases}1 & \text { if } 1+2 k^{*} N \leq s \leq N+2 k^{*} N \\
0 & \text { otherwise }\end{cases}
\end{aligned}
$$




$$
A_{j}(s-1)=\sum_{m=1+p}^{s-2} B_{j}(m) \frac{y_{m, s-1}}{\sum_{r=m}^{1+p+N} y_{m, r}} \quad s>1
$$

where $p=2 k * N+(1-\beta) N$

The occupancy $M_{j}(s)$ of the bus $j$ during the segment between stops $s-1$ and $s$ can be evaluated by Equation (14) taking into account the vehicle capacity, $C$. For formulation consistency, we state that $M_{\mathrm{j}}(0)=0$. Equation (15) evaluates the total amount $D_{j}(s)$ of waiting passengers at stop $s$ that cannot get on the bus $j$ (if they exist) and may board on the following bus $j+1$. It is supposed that the definition of the targeted headway $H$ (input of this model) is properly defined in order to accommodate the passenger demand in the static system with buses of capacity $C$.

$$
\begin{gathered}
M_{j}(s)=\min \left\{C ; M_{j}(s-1)+B_{j}(s)-A_{j}(s)+D_{j-1}(s)\right\} \\
D_{j}(s)=\max \left\{0 ; M_{j}(s-1)+B_{j}(s)-A_{j}(s)-C\right\}
\end{gathered}
$$

\subsection{Bus headway control strategies}

All strategies aimed at controlling fixed bus intervals are based on the real-time headway monitoring of bus departures from stops. Equation (16) evaluates the real time headway between two consecutive buses ( $\left.\Delta t_{j}^{d}(s)\right)$ considering the departure time from stop $s$. Therefore, it is necessary that each time any bus $j$ is going to depart from one stop $s$, the variable $\Delta t_{j}{ }^{d}(s)$ is updated. It should be noted in Equation (16) that the real-time evaluation of current headway of bus $j$ is made with regard to the bus $j-1$ ahead, at stop $s$ (forward comparison). This information should be used to calculate the adherence with regard to the targeted headway $H$ (see Equation 17a).

The headway analysis of bus $j$ with regard to bus $j+1$ (backwards) is infeasible because bus $j+1$ has not arrived yet at stop $s$. Hence, the backward comparison of headway with the following bus (Equation $17 \mathrm{~b}$ ) will be made taking into account the difference of departure time of bus $j$ and $j+1$ at the last stop $s^{*}$ visited by bus $j+1$ $\left(\Delta t_{j+1}{ }^{d}\left(s^{*}\right)\right)$ up to this moment.

$$
\Delta t_{j}^{d}(s)=t_{j}^{d}(s)-t_{j-1}{ }^{d}(s) \forall s
$$

forward comparison to bus $j-1$

$$
\begin{array}{r}
\varepsilon_{j-1, j}(s)=\Delta t_{j}^{d}(s)-H \\
\varepsilon_{j, j+1}\left(s^{*}\right)=\Delta t_{j+1}^{d}\left(s^{*}\right)-H
\end{array}
$$$$
\text { backward comparison to bus } j+1
$$

\subsection{Control strategies}

The model developed in section 2.1 only considers the usual practice of bus agencies, defined here as Strategy $S O$. It consists of the allocation of slack time $\phi_{s}$ at the holding points (terminals) in order to tackle the lack of regularity. However, as is reported in Daganzo (2009), this solution presents several problems. The control strategy is not adaptive, since slack times are not dependent to the deviation of targeted time headways. In fact, these slacks represent an unproductive allocation of time in the cycle time of buses when the performance of the system is regular.

Therefore, this paper analyzes two complementary fleet management strategies that may overcome the limitations of the previous operation. On one hand, Strategy S1 will be based on "dynamic holding points", so that the cruising speed of buses will be varied depending on the time-headway between buses. The control scheme of this strategy is practically similar to that presented in Daganzo (2009). On the other hand, Strategy $S 2$ presented here will encompass the previous variable bus speed pattern combined with an additional measure based on signal priority for buses. 


\section{Strategy S1}

This strategy obliges drivers to adapt the cruising speed of their bus when the headway adherence is irregular. The motion law that modifies the speed of bus is defined by Equation (18) when the vehicle capacity constraint of vehicles ahead and at rear are not achieved. Therefore, this formulation is only valid when $M_{j+1}\left(s^{*}\right)<\varphi C$ and $M_{j-1}\left(s^{\prime}\right)<\varphi C$, where $\varphi \cong 1$ and $s^{\prime}$ is the last visited stop of vehicle $(j-1)$ in the route.

$v_{j}(s)=\left\{\begin{array}{l}\frac{l_{s}}{l_{s} / v_{j}(s-1)+f_{f}\left(\varepsilon_{j, j+1}-\varepsilon_{j-1, j}\right)} \\ \min \left\{v_{b} ; \frac{l_{s}}{v_{s} / v_{j}(s-1)+f_{b}\left(\varepsilon_{j, j+1}-\varepsilon_{j-1, j}\right)}\right\}\end{array}\right.$

$$
\begin{aligned}
& \text { if } \varepsilon_{j, j+1}>\varepsilon_{j-1, j} ; \varepsilon_{j, j+1}>0 \\
& \text { if } \varepsilon_{j-1, j}>\varepsilon_{j, j+1} ; \varepsilon_{j-1, j}>0 \text { and } l_{s} / v_{j}(s-1)+f_{b}\left(\varepsilon_{j, j+1}-\varepsilon_{j-1, j}\right)>0 \\
& \text { otherwise }
\end{aligned}
$$

$v_{j}(s)=v_{b} \quad$ if $M_{j-1}\left(s^{\prime}\right) \geq \varphi C$ or $M_{j+1}\left(s^{*}\right) \geq \varphi C$

The first case of Equation (18) reduces the actual cruising speed when: i) bus $j$ is getting further away from bus $j+1$ than the desired headway $H$ (i.e. $\varepsilon_{j, j+l}\left(s^{*}\right)>0$ ); and ii) this time spacing is greater than the corresponding value with the vehicle ahead at stop $s$. It is desirable that this bus $j$ will operate the stretch up to the next station at a cruising speed below the maximal value in order to reestablish the desired time headway. The parameter $f_{f}$ is a speed adjusting factor $\left(f_{f}>0\right)$. Therefore, the speed reduction of vehicle $j$ is proportional to the difference between the total headway deviation and the vehicle at rear and ahead $\left(\varepsilon_{j, j+1}-\varepsilon_{j-1, j}\right)$.

On the other hand, the second case of Equation (18) increases the current cruising speed when: i) bus $j$ presents a higher headway with bus $j-1$ than the target value $H$ in stop $s$ (i.e. $\varepsilon_{j-1, j}(s)>0$ ); and ii) this headway is higher than the corresponding with the vehicle at rear at stop $s^{*}$. The reason is that bus $j$ will find more passengers at stops than the expected (increase in dwell time). These additional passengers would be supposed to get on bus $j+1$ if bus regularity would be perfect. If no control measures are implemented, one may suppose that this tendency will be amplified until bus $j+1$ reaches bus $j$ (bus pairing phenomena). In order to tackle this problem, it is recommended that bus $j$ will run at a higher speed than the previous segment $v_{j}(s)>v_{j}(s-1)$. As defined in Equations (18), the speed modifications are proportional to the difference of the time headway adherence between buses $(j, j+1)$ and buses $(j-1, j)$. Note that this difference $\left(\varepsilon_{j, j+1}-\varepsilon_{j-1, j}\right)$ has a negative value in the second case of Equation (18), where $f_{b}\left(f_{b}>0\right)$ is the speed adjusting factor in this situation. If we set a value of the speed adjusting parameter $f_{b}$ that produces $L /\left(v_{j}(s-1)\right)+f_{b}\left(\varepsilon_{j, j+1}-\varepsilon_{j-1, j}\right) \leq 0$; the corresponding cruising speed will present a negative value too. That possibility is constrained in the second case of Equation (18) since the system will require a higher cruising speed and de facto we will use $v_{j}(s)=v_{b}$ in these situations.

As it is pointed out in Daganzo (2009), the dimensionless speed adjusting parameters $f_{f}$ and $f_{b}$ represent the marginal increase in expected bus delay caused by a unit increase in headway. It may be considered as the expected number of passenger arrivals at one stop during the average marginal delay induced by one boarding move. Therefore, $f_{f}$ is also conceived as a speed factor to reestablish the desired headway between two consecutive buses. If $f_{f}=1$, the current headway will be close to the targeted headway in the next stop, but it will produce a significant reduction of bus speeds. Otherwise, if $f_{f} \rightarrow 0$, it will maintain the modified speed close to $v_{b}$ and it will take a great number of stops to overcome the deviation from desired headway. Similar statements can be provided for the adjusting parameter $f_{b}$.

However, when the occupancy of the bus ahead or at rear of bus $j$ is equal or slightly lesser than the vehicle capacity, it is preferable that bus $j$ runs at the maximal speed as it is defined in Equation (19). The reason of this statement is justified as the vehicle ahead or at rear will also present a passenger load similar to the vehicle capacity. It will experience shorter dwell times at stops since boarding operations are not made. Therefore, it will run at a maximal speed $v_{b}$ (it will follow the 3rd case of Equation 18). 
This strategy improves previous protocols aimed at maintaining regular time headways in two major aspects. Firstly, the speed reduction of Equation (18) is only activated for irregular arrival periods so that the production of the rest of the service is made at maximal speed. Secondly, the modification of bus speeds is adaptive to the range of deviation of time headways, overcoming the fixed holding points of the common practice of bus agencies. However, the speed reduction of those buses delayed to keep regular headways may suppose a reduction of the average commercial speed of the bus line. In fact, all strategies in this field presents a trade-off between speed (travel time) and regularity objectives. Therefore, the presented strategy of this section should be complemented with several operational actions aimed at increasing the speed of those delayed buses. Hence, bus agencies may tackle the regularity problems without affecting the average commercial speed of the line or maintaining idle times in the service.

\section{Strategy S2}

This new strategy essentially increases the commercial speed of the delayed vehicles, providing activated traffic light priority at intersections. It also encompasses the modification of speed proportional to $\varepsilon_{j, j+1}-\varepsilon_{j-1, j}$ (reduction or increase) proposed in strategy $S 1$ through the Equations (18) and (19). Here, the time headway adherence can be also tackled by the elimination of several vehicle stops at signalized intersections, due to a time extension of the green phase. This measure is the only strategy that increases the commercial speed of buses in the route. The variable $G$, expressed in units of time, represents a little extra green time introduced in every intersection (if necessary) in order to avoid the stop of a delayed vehicle at a traffic light section. If the arrival time of delayed bus $j$ at intersection $p$ is estimated during an interval $G$ after the end of the green phase, the TCC can keep the green phase a total amount of $g+G$ seconds. Since the traffic light cycle time is significantly lower than the time headway $\left(C_{p}<H\right)$, the TCC may revert and even truncate the available green time in the following cycle $(g-G)$. The reason is to guarantee the evacuation rate of the intersection and alleviate traffic or pedestrians queues. Therefore, high values of $G$ are not considered $\left(G \leq 0.4 C_{p}\right)$

The activation of the green extension is only permitted when bus $j$ presents time headways with regard to the vehicle ahead (advanced) higher than the target headway and the actual headway with the vehicle at rear. It is equivalent to the situation when cruising speed in Strategy S1 is evaluated through the second case of Equation (18). Under those circumstances, the bus travel time in the segment $(s ; s+1)$ may be even reduced due to soft modifications of red phase at the intersections located along the segment. To do so, it is necessary to verify the state of the traffic light phases when delayed buses arrive at intersections in order to activate the green extension strategy. The first case of Equation (20) determines the departure time of vehicle $j$ from intersection $p$ when the green extension is activated. It replaces Equation (5) to describe the vehicle motion law under the implementation of this regularity controlling strategy. The right condition of the first case of Equation (20) represents that vehicle $j$ arrives within a green phase of $g+G$ length considering the initial offset $\Delta_{p}$. When this constraint is not accomplished, the vehicle must wait until the red phase in the traffic light is finished (second case of Equation 20).

$t_{j, p}{ }^{d}= \begin{cases}t_{j, p}{ }^{a} & \text { if } t_{j, p}{ }^{a}<\left(\left(C_{p} n_{j p}{ }^{*}\right)+\Delta_{p}+(g+G)\right) \\ C_{p}\left(n_{j p} *+1\right)+\Delta_{p} & \text { in other cases }\end{cases}$

\section{EVALUATIONS}

The bus motion law and the controlling strategies explained in the last section have been programmed into a simulation tool. Some performance indicators have been defined to measure the time headway adherence, the travel time of passengers and the operating cost to provide the service under each controlling strategy. Moreover, three set of bus problems have been generated to assess the system performance when a control strategy is activated to overcome headway variation. The first set is based on an idealized bus route of 40 stops evenly distributed. There are three key parameters that will be modified: the capacity of vehicles, the bus disturbance and the passenger demand. The second set of problems embraces different traffic signal settings and number of intersections along the corridor. Eventually, the third problem represents a real route of Barcelona's high performance bus network. All the information concerning bus stops, traffic light management and traffic behavior has been collected from campaigns or provided by both the bus agency and city council. 


\subsection{Performance Indicators Definition}

Four metrics are proposed to assess the quality of the solutions associated to each control strategy. One of the objectives of the operator manager is to provide a fast service in order to minimize the time spent in the system by the passengers. It is evaluated by means of the first indicator, the total passenger travel time (Equation 23) which includes the in-vehicle travel time (Equation 21) and waiting time (Equation (22) of all passengers in the system (excluding access and egress time).

$$
\begin{gathered}
T T=\sum_{j=1}^{J} \sum_{s=2 N+1}^{\left(m^{*}+\alpha_{j}\right)(2 N)}\left(t_{j}^{a}(s+1)-t_{j}^{a}(s)\right) M_{j}(s)+\max \left(\gamma B_{j}(s) ; \delta A_{j}(s)\right)\left(M_{j}(s)-A_{j}(s)\right) \\
T W=\sum_{j=1}^{J} \sum_{s=2 N+1}^{\left(m^{*}+\alpha_{j}\right)(2 N)}\left(\frac{q}{2}\left(t_{j}^{a}(s)-t_{j-1}^{a}(s)\right)^{2\left(2+2 k^{*-\beta}\right) N} \sum_{r>s} y_{s r}+D_{j}(s)\left(t_{j}^{a}(s)-t_{j-1}^{a}(s)\right)\right) \\
T P T=T T+2.2 T W \\
T P T \text { (in pax-hours) }
\end{gathered}
$$

The estimation of total travel time through the Equations (21)-(23) will be made during a temporal period of analysis that has been defined as a predefined number of vehicles departures $D$ from stop $s=2 N+1$. It refers to the initial stop in direction A-B but once each vehicle has completed one round trip to warm up the system. If the analysis had been done for a given period of time ( 2 hours for example), the number of on-board passengers would have been lower for highly unstable bus routes, where several vehicles would have moved in platoons. Therefore, the first indicator would have presented lower user cost in those situations than in perfect regularity lines, which is obviously incorrect. Let $m^{*}$ be the number of completed round trips by all fleet $\left(m^{*}=[D / J]^{+}\right)$ when $D$ vehicle departures would be considered to evaluate $T T$ and $T W$ variables. The first $\left(D-m^{*} J\right)^{\text {th }}$ vehicles will have run an additional round trip. Parameter $\alpha_{\mathrm{j}}$ is equal to 1 if bus $j \leq\left(D-m^{*} J\right)$ and 0 otherwise to account for this additional round trip. In Equation (22), the first term within brackets captures the waiting time of passengers that have arrived at stop $s$ after the boarding process of bus $j-1$. As we consider a constant arrival rate of passengers at stop, it is calculated as one half of the current time spacing between buses $(j ; j-1)$ multiplied by the number of passengers arrived within this interval. The latter is addressed as the product of the passenger flow in one direction $(q)$, the flow percentage between stop $s$ and all potential destinations stops $r\left(y_{s r}\right)$ and the lapsed time between the arrival of bus $j$ and $j-1$. The second term considers the passengers arrived at stop $s$ before the arrival of bus $(j-1)$ that could not get on this bus (or even previous buses) due to capacity constraint. The total amount of passengers under this situation $\left(D_{j}(s)\right)$ must wait the time period between the arrival of consecutive buses $(j, j-1)$. In fact, $D_{j}(s)$ is updated at each stop $s$ for each bus $j$ considering the boarding capacity of the predecessor buses. In Equation (23), the total passenger waiting time is multiplied by a factor of 2.2 in order to magnify the higher perception of waiting time by users related to in-vehicle travel time. This value is consistent to the factors proposed in TRB (2013) and Hill (2003), which range from 2.1-2.8 times the value of in-vehicle time.

The second indicator is the operating cost of the bus system in the period under analysis $\left(Z_{O}\right)$, defined in Equation (24). It is estimated as the product of the total vehicle hours in service by the unit temporal cost of one vehicle $\left(c_{t}\right.$, in terms of $€ /$ veh-h). The hours in service by the whole fleet are estimated as the difference between the arrival times at the end of the route in the $\left(m^{*}+\alpha_{\mathrm{j}}\right)^{\text {th }}$ and first roundtrip.

$$
Z_{O}=c_{t} \sum_{j=1}^{J}\left[t_{j}^{a}\left(\left(m^{*}+\alpha_{j}\right) 2 N\right)-t_{j}^{a}(2 N+1)\right] \quad \text { (in Euros) }
$$

The third indicator proposed is the total cost of the system $\left(Z_{T}\right)$, including the operating cost and the temporal cost incurred by passengers. As control strategies present a trade-off between reducing bus headways variations and increasing operating cost, this metric will capture which strategy is more cost-efficient regarding the others. Equation (25) defines how this variable is evaluated based on the former indicators and the passenger value of time, $\mu(€ /$ pax-h).

$$
Z_{T}=Z_{O}+\mu \cdot T P T \quad \text { (in Euros) }
$$

The last metric is the coefficient of headway variation. It is defined as the sum of the variation in time 
between the arrival of one bus and the arrival of the next bus at a stop, divided by the average time headway in the period of analysis. It is necessary to point out that the overall headway alterations in our model take place when the first bus is currently serving the second cycle. The first cycle is only used to introduce uniformly the whole vehicles in the route, in other words to warm up the simulation. By this reason, the time headways between buses in the first cycle are not taken into account. In TRB (2013), Equation (26) is proposed to evaluate the average headway variation over all stops,

$$
c_{v}=\frac{S}{\bar{h}} \geq 0
$$

where $c_{v}$ is the coefficient of headway variation, $S$ the standard deviation of all headways observed in all potential stops during the complete tracking of $D$ departures from the stop $s=2 N+1$ (first round cycle is excluded as we suppose that the system is under a perfect time-headway adherence) and $\bar{h}$ the average headway. In TRB (2013), the level of service ( $\mathrm{LoS})$ concerning time-headway adherence is ranged considering the following domains: $\mathrm{A}\left(c_{v} \leq 0.21\right), \mathrm{B}\left(0.21<c_{v} \leq 0.3\right), \mathrm{C}\left(0.3<c_{v} \leq 0.39\right), \mathrm{D}\left(0.39<c_{v} \leq 0.52\right), \mathrm{E}\left(0.52<c_{v} \leq 0.74\right)$ and $\mathrm{F}$ $\left(c_{v} \geq 0.75\right)$.

\subsection{Problem Generation}

The first set of problems is composed by three test instances that differ in the key parameters, playing a significant role in headway control techniques. These parameters are the passenger flow ( $q=1000$ or $1400 \mathrm{pax} / \mathrm{h}$ in each direction), a homogeneous vehicle capacity ( $C=75$ or unlimited) and the total amount of time disturbance that any occasional event may cause in a specific bus ( 2 or 4 minutes). A list of values for other common parameters in the first set of problems is summarized in Table 1. Problem Set 2 is aimed at analyzing the behavior of the control strategies for different traffic signal settings.

The estimation of the boarding and alighting passenger flow at each bus stop for each direction is made considering a stationary passenger flow matrix between stops $(o, d)$ during the whole analysis, $Y_{o d}$, where $o=1, .$. , $N-1 ; d=o+1, . . N$ in direction A-B; $o=N, . .2 N-1 ; d=o+1, . ., 2 N$ for direction B-A. This matrix is estimated by the product of the former passenger flow in the line $(q)$ and the percentage of passenger flow matrix $y_{o d}$ between stops. The latter is considered to be constant in the Problem Set 1 and it is evaluated from Table 2. It is supposed that the O-D distribution of trips is equal in both route directions (A-B, B-A). The door opening and closing time $t_{o c}$ is neglected in this set of problems.

For simplicity, we assume that stops are evenly distributed along the route in Problem Set 1 and 2 . These problems are constituted by 40 stops ( $N=20$ in each direction) uniformly distributed every 300 meters along the route. In Problem Set 1 , the intersections are located every $l=150$ meters while in Problem Set 2 the intersection spacing is $l=\{100 ; 210 ; 300\}$ meters. It is assumed that the first intersection $(i=1)$ is $x_{o}=l / 2$ meters away from the stop $s=1$ (origin of coordinates). The signal offsets of the overall intersections are calculated to avoid the stop of private vehicles along the corridor, i.e. a "green wave" is guaranteed for cars. If the average car speed is $v_{c}$ $(\mathrm{m} / \mathrm{s})$, it is possible to infer the initial time of green phase $t_{g, i}$ at intersection $i$ by Equation (27):

$$
t_{g, i}=\frac{x_{o}+l(i-1)}{v_{c}} \quad i=2, . ., I
$$

In the Problem Set 1 and 2, it is supposed that the deviation $U_{j}(s)$ from the time-headway takes place at vehicle $j=2$ at stop $s=42\left(U_{2}(42) \geq 0\right.$; otherwise $\left.U_{j}(s)=0\right)$. The stop location has been chosen for two reasons: to allow all vehicles to complete one round trip in the bus route (to warm up the system); its occurrence is far away enough from a holding point (located at $\operatorname{stop} s=60$ ) so that the deviation will propagates up-stream and downstream during a large part of one direction of the service. 
TABLE 1. Input parameters in the modeling of Problem set 1, 2 and Problem 3

\begin{tabular}{|c|c|c|c|c|c|}
\hline \multirow[t]{2}{*}{ Concept } & & & \multicolumn{3}{|c|}{ Value } \\
\hline & \multicolumn{3}{|c|}{ Problem Set 1} & Problem Set 2 & Problem 3 \\
\hline Maximal cruising speed, $v_{b}(\mathrm{~m} / \mathrm{s})$ & \multicolumn{3}{|c|}{10} & 10 & 13.88 \\
\hline Number of bus stops in the round trip ( $2 \mathrm{~N})$ & \multicolumn{3}{|c|}{40} & 40 & 39 \\
\hline Boarding time per passenger, $\gamma(\mathrm{s})$ & \multicolumn{3}{|c|}{3} & 3 & 3.7 \\
\hline Alighting time per passenger, $\delta(\mathrm{s})$ & \multicolumn{3}{|c|}{2} & 2 & 2.1 \\
\hline Targeted time headway, $H(\mathrm{~s})$ & \multicolumn{3}{|c|}{300} & 300 & 300 \\
\hline Stop when disturbance takes place & \multicolumn{3}{|c|}{$\# 2(\# 42)^{\mathrm{a}}$} & $\# 2(\# 42)^{\mathrm{a}}$ & -- \\
\hline Vehicle affected by time disturbance & \multicolumn{3}{|c|}{ \#3 } & \#3 & -- \\
\hline Intersection spacing, $l(\mathrm{~m})$ & \multicolumn{3}{|c|}{150} & $100-300$ & \\
\hline Traffic light cycle time, $C_{p}(\mathrm{~s})$ & \multicolumn{3}{|c|}{100} & 90 & Var. \\
\hline Green phase time, $g(\mathrm{~s})$ & \multicolumn{3}{|c|}{50} & $22.5-67.5$ & Var. \\
\hline Green extension in strategy $\mathrm{S} 2, G(\mathrm{~s})$ & \multicolumn{3}{|c|}{$5-20$} & $5-20$ & $5-20$ \\
\hline Door opening and closing time, $t_{o c}(\mathrm{~s})$ & \multicolumn{3}{|c|}{0} & 0 & 2 \\
\hline \multirow[t]{3}{*}{ Layover time, $\theta_{A}, \theta_{B}(\mathrm{~s})$} & \multicolumn{3}{|c|}{180} & 180 & 180 \\
\hline & Problem & Problem & Problem & & \\
\hline & 1.1. & 1.2 & 1.3 & & \\
\hline Vehicle capacity, $C$ (pax/veh) & unlimited & 75 & 75 & 75 & 134 \\
\hline $\begin{array}{l}\text { Hourly Passenger flow in direction A-B and } \\
\text { B-A, } q(\text { pax/h) }\end{array}$ & 1000 & 1000 & 1400 & 1400 & 1400 \\
\hline Time disturbance, $U(\mathrm{~s})$ & 120 & 120 & 240 & 240 & -- \\
\hline
\end{tabular}

${ }^{a}$ The term in brackets denotes that disturbance takes place once vehicle has completed one round trip (40 stops) before visiting stop \#2

\section{TABLE 2 a) Matrix $y_{o d}$ (percentage of passengers trip among stops); b) Percentage of boarding and alighting passengers}
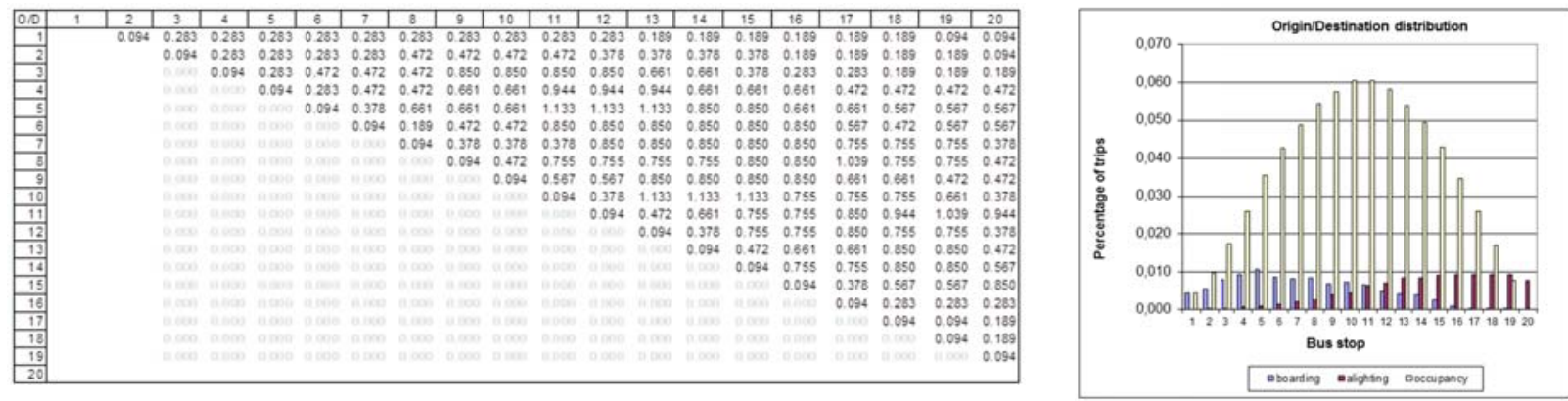

The implementation of strategy $S 0$ considers two different slack time settings of $\phi_{s}=3$ and 6 minutes at each holding point of the route. The holding points are located at the ending stop of each route direction (terminals or headers) where it is supposed that no passengers are on board the vehicles. This fact means that different number of resources will be needed to operate the service depending on the value of slack time $\left(\phi_{s}\right)$ provided in the route, as it is determined in Equation (1). We enumerated of the total system cost and the coefficient of headway variation for different combinations of values of $f_{f}, f_{f}$. The values considered for both parameters were $f(n)=0.0001 \cdot\left\{2^{\bmod (n, 2)} \cdot 0.5 \cdot 10^{[n / 2]^{-}}\right\}$for $n=1, . .9$. The mathematical operator $\bmod (x, y)$ defines the remainder after $x$ is divided by $y$; whereas $[x]^{-}$rounds $x$ to the lower nearest integer number of $x$. We found out that the pair of parameter values that minimized the total cost and headway variations in the implementation of Strategy $S 1$ in Problem 1.1 were $f_{f}=0.01, f_{r}=0.05$. Therefore, the evaluation of strategy $S 1$ and $S 2$ is made in the whole problems considering $f_{f}=0.01, f_{r}=0.05$ and a vehicle capacity threshold of $\varphi=0.95$ in Equation (19). Finally, the results obtained with the previous strategies are compared with the corresponding of strategy $S 2$, in which the green phase extension is chosen to be $G=5,10$ and 20 seconds. Moreover, the results provided by the implementation of strategy S1 and S2 together with a slack time $\phi_{s}=3$ min at the headers (holding point) are also assessed.

The results provided by each controlling strategy (SO, S1 and $S 2)$ in Problem Set 1 and 2 can be compared to a Baseline case in which no alteration is considered $(U=0)$. This case represents a perfect time- 
headway adherence among buses and regular bus arrivals at stops, so that no controlling strategy is needed. In addition to that, we also assessed the performance of the route under the time disturbance when no controlling strategy is implemented and the system is totally uncontrolled (Uncontrolled case). In the modeling framework, this case is equivalent to the implementation of strategy $S 0$ when $\phi_{s}=0 \mathrm{~min}$.

The other test instance considered in this paper (Problem 3 in Table 1) is the route with highest demand of Barcelona local bus network (H6 line). This straight-shaped line connects several university campus and new business areas to residential districts, running along quite congested streets and avenues (Figure 3). The line presents a target time headway of $H=5 \mathrm{~min}$ and a total passenger flow of $q=1400 \mathrm{pax} / \mathrm{h}$ in the period of study. The line has a mandatory layover time of 3 minutes and an additional slack time of several minutes to tackle bus bunching ( $\phi_{s}=1,3$ and $6 \mathrm{~min}$ ), with 22 buses operating the roundtrip service. The capacity of these buses is 134 pax/veh. The line is $19.3 \mathrm{~km}$ long with 39 bus stops. Moreover, in this example, the unit boarding and alighting time are respectively $\gamma=3.7 \mathrm{~s} /$ pax and $\eta=2.1 \mathrm{~s} / \mathrm{pax}$; while the opening and closing door time is $t_{o c}=2 \mathrm{~s}$. The maximal speed in the bus lane is considered to be $v=50 \mathrm{~km} / \mathrm{h}$. These data were determined taking into account average values from the real operation of this line reported by TMB (major bus operator of Barcelona). The details of the traffic light settings are summarized in the Appendix.

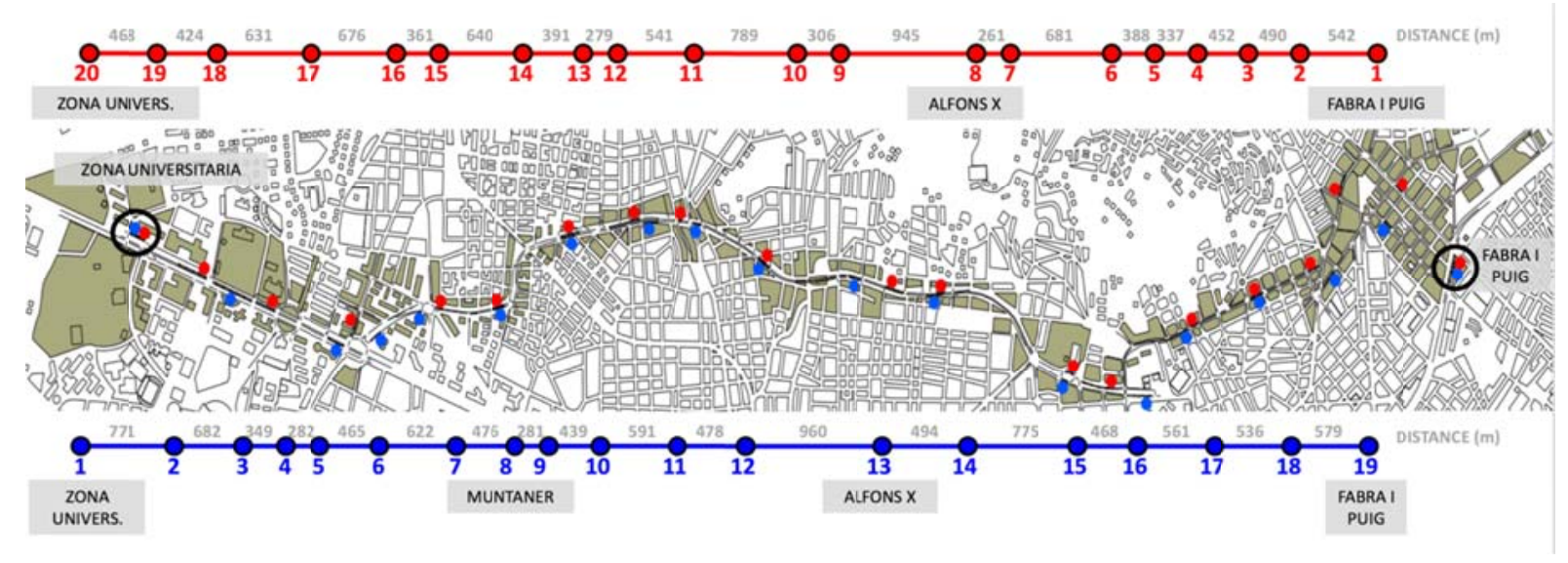

\section{FIGURE 3 Layout of the bus route $\mathrm{H6}$ operated by TMB}

The simulation of this instance has been developed through the software Aimsun ${ }^{\circ}$, which includes a microscopic simulator of traffic and transit systems. This software allows us to analyze the performance of bus route under the influence of other traffic (private cars) and taking into account the real synchronization of traffic lights along the corridor. The information about (car) traffic flows in the streets and the traffic lights parameters is provided by the Barcelona City Council- Mobility Department. Moreover, the activation of strategy $S 1$ and $S 2$ is only considered when $\varepsilon_{j-1, j}(s)>E$, $(E \geq 0)$. It means that the modification of bus speeds and traffic lights offsets is only activated if the time headway deviation is greater than a target threshold $E$. The potential values considered are $E(\mathrm{sec})=\{0 ; 15 ; 30\}$.

\subsection{Results and Discussion}

The results obtained by the former control strategies are compared independently for each problem set. Problem Set 1 encompasses several idealistic instances where the target bus headway was a multiple of the traffic signal cycle time $\left(H / C_{p}\right.$ is an integer value). It means that for the baseline case where no exogenous disturbance is considered $\left(U_{j}(s)=0 \forall j, s\right)$, all vehicles present the same roundtrip time and, consequently, the coefficient of headway variation will be $c_{v}=0$. Problem Set 2 is aimed at carrying out a sensitivity analysis about the effect of traffic signal setting on the performance of each strategy, especially for Strategy $S 2$. In these instances, we force that $H / C_{p}$ will not be an integer value. Vehicles will find different sequences of green-redl phases at intersections even in the baseline case; therefore $c_{v}>0$. Finally, the third problem represents a real instance of a high performance bus route in Barcelona, where each intersection presents a different signal setting from the others. 


\subsubsection{Unstable motion created by exogenous disturbances (Problem set 1)}

The results provided in Problem 1.1 with the implementation of each controlling strategy are summarized in Figure 4. The numerical analysis encompasses the whole round trips of $D=27$ vehicles departed from the original stop of the route. In an idealized system with no disturbances (base line case), the indicator values are TPT $=910$ hours, $\mathrm{Z}_{\mathrm{O}}=11,844 €, \mathrm{Z}_{\mathrm{T}}=25,499 €$ and $c_{v}=0$. The line segment between stops (\#11,\#12) presents the critical passenger load, $O_{\max }=50.44 \mathrm{pax} / \mathrm{veh}$. When we introduce a time disturbance $U=2 \mathrm{~min}$, the Uncontrolled case provides an enormous total user travel time, total cost as well as a high value of the coefficient of headway variation associated to bunching effect. In this case, as we suppose that vehicles have unlimited capacity, any bus suffering a delay will be able to serve all waiting passengers in the following stops of the route. Hence, the commercial speed of this bus drops due to increasing dwell times at stops. It will be considered as a movable bottleneck since overtaking is not allowed. At the end of simulation, buses move in platoons of 5 vehicles. Therefore, the total travel time of the users is basically constrained by the commercial speed of that bus. The disturbance propagates along the route with no control. The variable TPT is 187 times greater than the corresponding value for the situation in which no disturbance occurs (base line case). As some slack time is introduced, the user costs, agency cost and regularity are partially improved. Although a slack time $\phi_{s}=3$ is provided at each header in strategy $S O$ (greater than the disturbance $U=2 \mathrm{~min}$ ), the system still presents a high total travel time, operating and total cost $\left(T P T=30,678\right.$ hours, $Z_{\mathrm{O}}=85,567$ Euros and $\left.\mathrm{Z}_{\mathrm{T}}=495,256 €\right)$. In fact, the total cost is 19.4 times greater than the baseline case. Only when the slack time is $\phi_{s}=6 \mathrm{~min}$, the user and agency costs are comparable with the results of the base line case. The time headway adherence is significant since the value $c_{v}=0.17$ corresponds to a level of service A according to TRB (2013). The control of system regularity with strategy $S O \phi_{\mathrm{s}}=6 \mathrm{~min}$ is made at expenses of deploying 3 additional buses (compared to the base line, $J=9$ vehicles). However, the total cost of the system is only 1.12 times greater than the baseline. This fact reflects the non-scalable and adapting nature of this strategy. While the slack time of $\phi_{\mathrm{s}}=3 \mathrm{~min}$ was not sufficient to prevent the propagation of bus disturbances, strategy $S 0$ with $\phi_{\mathrm{s}}=6 \mathrm{~min}$ can maintain a proper level of service and total cost compared to the base line case. Bus operation controllers do not know in advance the potential propagation; hence the slack time definition will be made blindly.

Strategy $S 1$ is able to significantly reduce the total user travel time by $82 \%$ compared to the Uncontrolled case $\left(S O, \phi_{s}=0\right)$. Strategy $S 1$ mitigates the increasing dwell times of a delayed bus by reducing the cruising speed of the vehicle ahead and at rear to maintain the targeted headway. It reduces the number of vehicles moving in bunches, but the performance of the line can be still considered negative. The total cost of the system is still 5.8 times greater than the base line case. The combination of strategy $S 1$ with the provision of minimal holding times $\left(S 1, \phi_{s}=3 \mathrm{~min}\right)$ outperforms the results given by Strategy S0 with $\phi_{s}=3 \mathrm{~min}$. Therefore, the provision of slack times can improve the behavior of Strategy $S 1$. However, the coefficient of headway variation $\left(c_{v}=0.79\right)$ is still unacceptable. Finally, the implementation of strategy $S 2$ improves the metrics of the service performance and costs $\left(T P T, \mathrm{Z}_{\mathrm{O}}, \mathrm{Z}_{\mathrm{T}}\right.$ and $\left.c_{v}\right)$ provided by strategy $S 1$ without slack times. Generally, all indicators can be enhanced as the green extension time $(G)$ is increased. It is noticeable that when the green extension time is set to $G=20$ seconds, the cost-oriented indicators are even less than the corresponding value for the base line scenario $\left(T P T=901.8\right.$ hours, $\left.\mathrm{Z}_{\mathrm{O}}=11,745 €, \mathrm{Z}_{\mathrm{T}}=25,254 €\right)$. However, the metric time headway adherence is now $c_{v}=0.17$, the same value obtained by strategy $S 0$ when $\phi_{s}=6 \mathrm{~min}$. Strategy $S 2$ maintains constant the number of vehicles that run the service ( $J=9 \mathrm{veh}$ ) whereas a slack time of $\phi_{s}=6$ min increases the fleet size to $J=12$ veh. For these reasons, we can state that strategy $S 2$ can guarantee the same level of service as static holding points (strategy $S 0$ ) without increasing the operating costs.

The analysis of the results in Problem 1.2 highlights the importance of the vehicle capacity in the headway control strategies. Figure 5 summarizes the main results considering the full round trip of $D=27$ departed vehicles. As it is the same bus route, the baseline scenario presents the same results of Problem 1.1.The metrics for the uncontrolled case $\left(T P T=1562 \mathrm{~h}, \mathrm{Z}_{\mathrm{O}}=13,170 €, \mathrm{Z}_{\mathrm{T}}=36,599 €, c_{v}=1.10\right)$ are significantly lesser than those obtained in Problem $1.1\left(T P T=170,341 \mathrm{~h}, \mathrm{Z}_{\mathrm{O}}=85,567 €, \mathrm{Z}_{\mathrm{T}}=2,640,687 €, c_{v}=4.19\right)$. For this problem, the uncontrolled case presents values of $T P T, Z$ o and $Z_{\mathrm{T}}$ that are, respectively, 1.7, 1.11 and 1.43 times greater than the metrics obtained in the baseline case. As delayed buses arrive at stops, the number of waiting passengers is steadily growing, but the boarding passenger operation is constrained. Therefore, when the capacity constraint is active, the dwell time of this vehicle drops significantly as it only allows passengers to alight. The unserved passengers at stops will wait for the next bus that will arrive with a shorter headway. Therefore, the activation of capacity constraint mitigates and stabilizes the headway variations without any controlling strategy. As a result, the commercial speed of the vehicle that suffers the disturbance is quite higher than the same vehicle in Problem 1.1. 


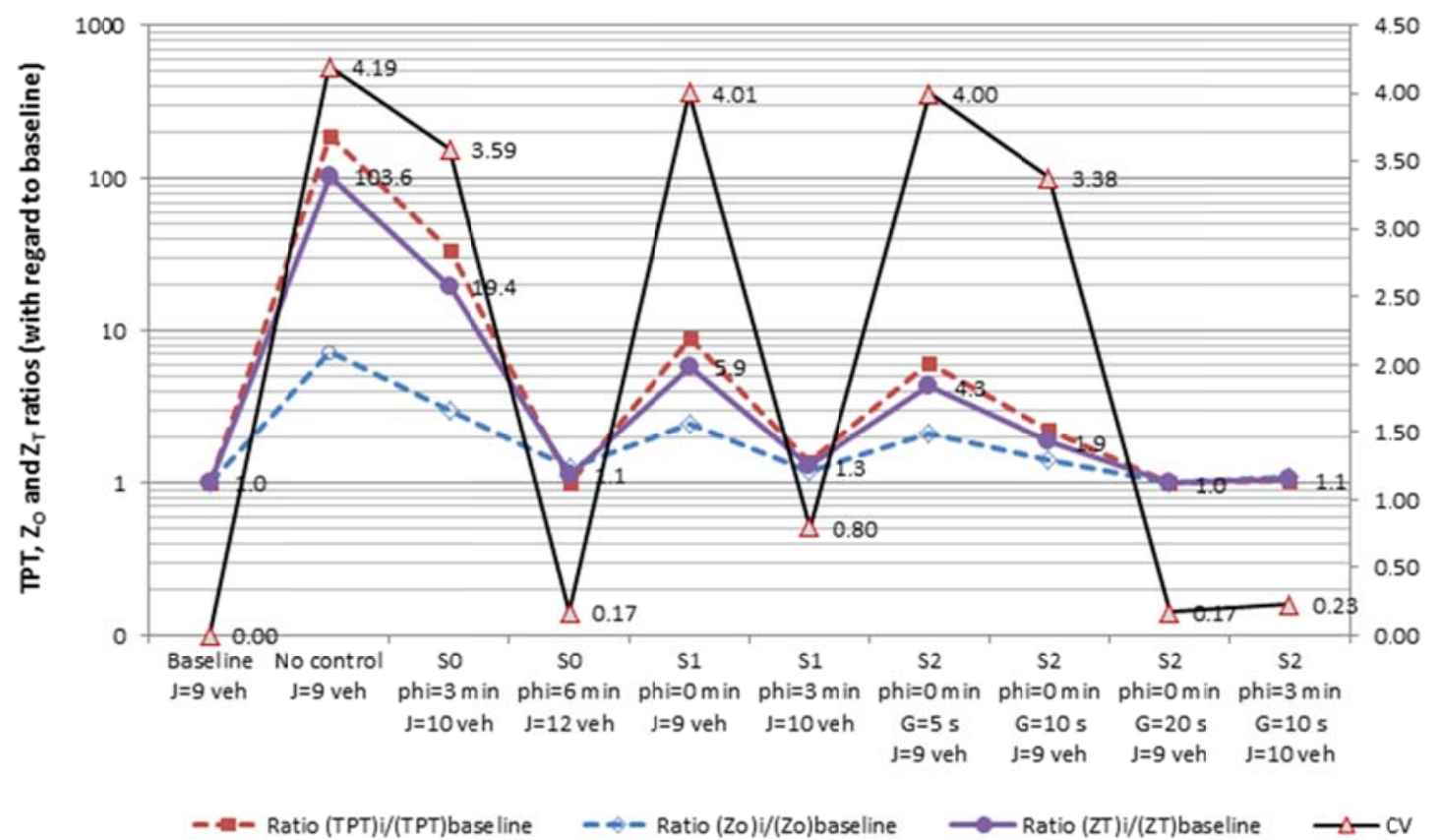

FIGURE 4. Total passenger travel time and coefficient of headway variation for each controlling strategy in Problem 1.1

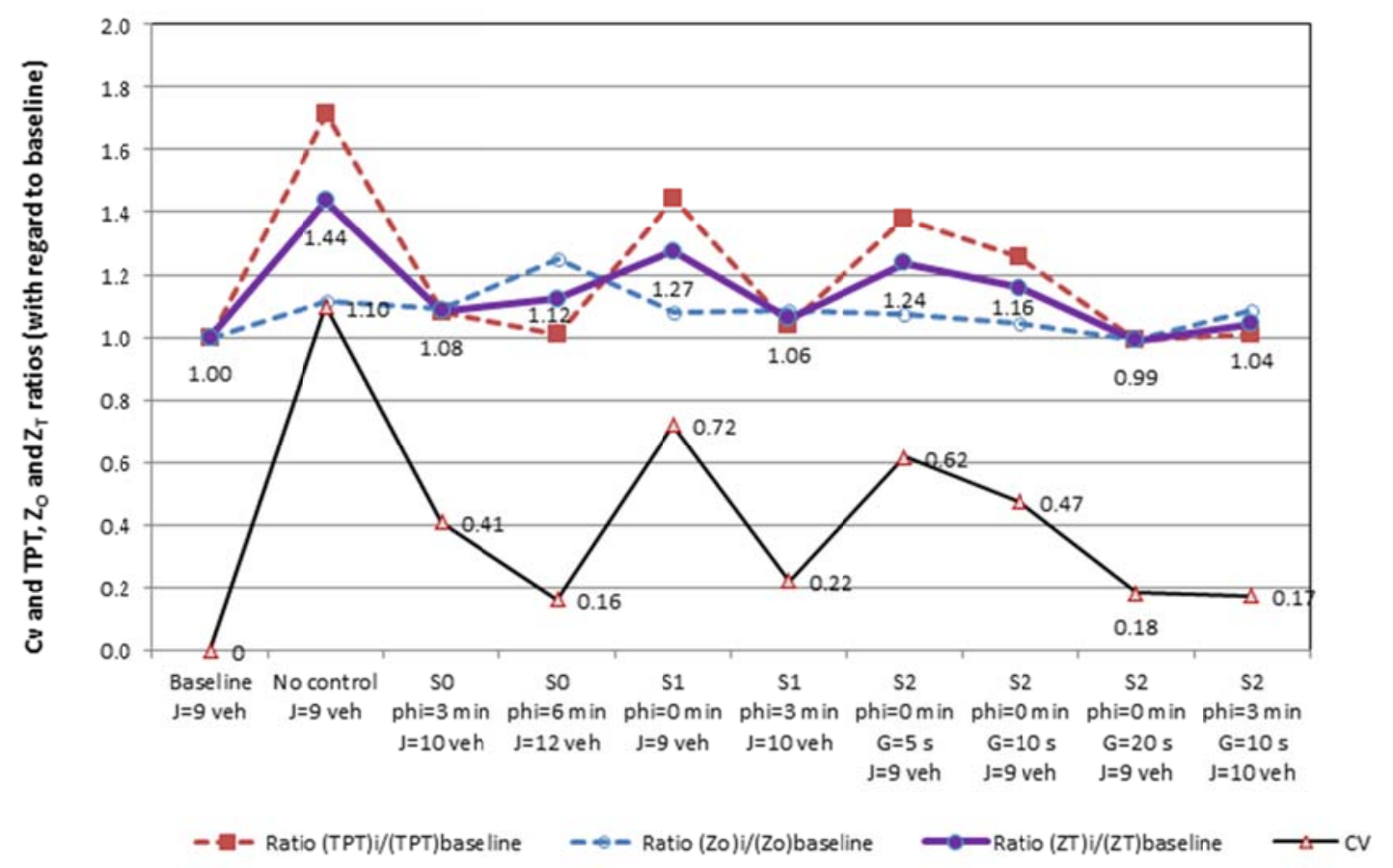

FIGURE 5. Total passenger travel time and coefficient of headway variation for each controlling strategy in Problem1.2

The provision of a slack times of $\phi_{s}=3 \mathrm{~min}$ (strategy $S 0$ ) to control the headways provides good results to compensate the time disturbance $U_{3}(42)=2 \mathrm{~min}$. The total cost is now 1.08 times the corresponding cost in the base line case. The extension of slack time to $\phi_{s}=6 \mathrm{~min}$ improves the user travel time in comparison to strategy So $\phi_{s}=3 \mathrm{~min}$ and reduces the headway variation up to $\mathrm{c}_{\mathrm{v}}=0.16$. However, the operating cost is increased due to 
the provision of 2 additional vehicles. Now, the strategy S0 with short slack times ( $\left.\phi_{s}=3 \mathrm{~min}\right)$ is more efficient than larger slacks $\left(\phi_{s}=6 \mathrm{~min}\right)$, in terms of total cost.

The total passenger time savings with implementation of strategy $S 1$ are not as important as in Problem 1.1 since the capacity constraint also helps to stabilize the performance ( $15 \%$ of improvement with regard to the Uncontrolled case). This fact is consistent with the statement in Muñoz et al. (2015) where it is said that the adaptive controlling speed proposed by Daganzo does not work in routes with high demand and where capacity constraint is not considered. The total cost of this strategy is 1.27 times the base line case, even higher than the corresponding figures of strategy SO. Additionally, the time headway adherence is not acceptable since it is graded as $\operatorname{LoS}$ E $\left(c_{v}=0.72\right)$. If we add a slack time of $\phi_{s}=3 \mathrm{~min}$, strategy $S I$ outperforms. strategy $S 0$, especially in the time headway adherence analysis whose metric is reduced to $c_{v}=0.22$. Finally, the implementation of strategy $S 2$ is able to compensate the passenger time lost due to disturbance and the natural motion of buses with travel time savings at the traffic lights. If the green extension time is minimal ( $G \leq 10$ seconds), the cost metrics are slightly higher than the baseline case. Nevertheless, this strategy outperforms the metrics provided by strategy $S 1$. However, as this green extension is determined to be $G>10$ seconds, the results are comparable to those of Strategy $S 0$ with slack times. If this traffic light controlling parameter is set to $G=20$ seconds, it produces the minimal total cost $\left(Z_{\mathrm{T}}=25,275 €\right)$. However, the coefficient of headway variation is slightly greater than the corresponding to strategy $S O \phi_{s}=6 \mathrm{~min}$, due to the discrete time saving at traffic lights. It is also remarkable that the hybrid controlling strategy ( $S 2$ with slack time $\phi_{s}=3 \mathrm{~min}$ ) gives good results (variable $Z_{T}$ is only 1.007 times greater than the base line and $c_{v}=0.17$ ) without imposing huge dynamic changes in traffic light management. The idea is to allocate a minimal slack time at headers (estimated as a function of an expected or recurrent disturbance value in the route) and mitigate larger disturbances performing strategy $S 2$ (variable cruising speed pattern and green traffic lights extension).

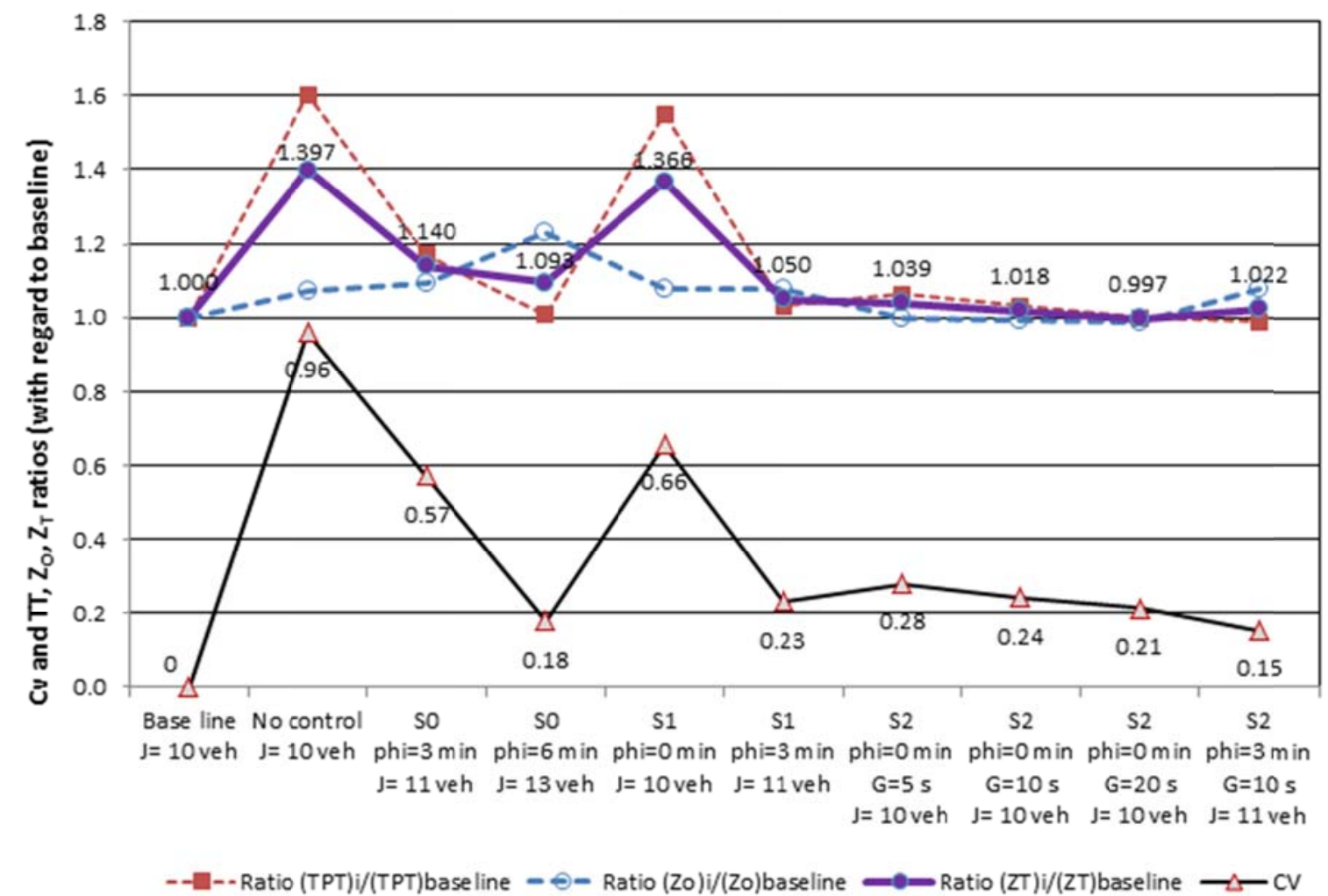

FIGURE 6. Total passenger travel time and coefficient of headway variation for each controlling strategy in Problem1.3.

Problem 1.3 is aimed at stressing the performance of the previous bus route with higher demand flow ( $q=1400 \mathrm{pax} / \mathrm{h}$ in both directions) and time disturbance $(U=4 \mathrm{~min})$. Figure 6 plots the results when the round trip of $D=30$ vehicle departures have been completed. The performance in the baseline case where no disturbance is considered is summarized by $T P T=1547 \mathrm{~h}, \mathrm{Z}_{\mathrm{O}}=14,701 €, \mathrm{Z}_{\mathrm{T}}=37,912 €, c_{v}=0$ and $J=10$ veh. The highest vehicle passenger load in the route $O_{\max }=70.56 \mathrm{pax} / \mathrm{veh}$ is detected between stops \#11 and \#12, and almost equals the vehicle capacity ( $C=75 \mathrm{pax} / \mathrm{veh})$. When the disturbance of $U=4 \mathrm{~min}$ occurs, when no controlling strategy is 
activated, the TPT, $Z_{\mathrm{O}}$ and $\mathrm{Z}_{\mathrm{T}}$ are increased respectively by $60 \%, 7 \%$ and $40 \%$ with regard to the baseline case. The time headway adherence reaches $c_{v}=0.96$. Strategy $S O$ is able to stabilize the system. When slack times are $\phi_{s}=6 \mathrm{~min}$ at terminals, the results in terms of total cost are quite similar to the baseline case $\left(\mathrm{Z}_{\mathrm{T}}\right.$ is increased by $9.3 \%)$ and the level of service of time headway adherence $\left(c_{v}=0.18\right)$ is stated as LoS A. In this problem, strategy S1 without slacks is not effective since the metrics are comparable to the corresponding values of the uncontrolled case. It is noticeable that only by reducing the speed of vehicles in systems with high demand, low vehicle capacity and short headways; we cannot maintain a good performance of the route. On the contrary, strategy $S 2$ outperforms the results of the previous controlling strategies. The total cost of the system $\left(\mathrm{Z}_{\mathrm{T}}\right.$ metric) is increased by $3.9 \%(G=5 \mathrm{sec}), 1.8 \%(G=10 \mathrm{sec})$ and $-0.3 \%(G=20 \mathrm{sec})$ compared to the baseline case. In these situations, the performance in terms of time headway adherence can be considered as LoS B. In this problem, the implementation of strategy $S 2$ with $G=10$ seconds and slack times $\phi_{s}=3$ min even improves the total travel time with regard to the baseline case and $c_{v}=0.15(\operatorname{LoS} \mathrm{A})$. However, the total cost is slightly greater than baseline case and strategy $\mathrm{S} 2$ with $\mathrm{G}=10$ s due to the inclusion of an additional vehicle.

\subsubsection{Unstable motion created by traffic lights and exogenous disturbances (Problem Set 2)}

A sensitivity analysis of the performance of each control strategy has been done in Problem Set 2 with regard to the traffic light settings. We considered three different intersection spacings along the route: $l=\{100 ; 210 ; 300\}$ meters. Moreover, we have also generated problems with different green time at signaled intersections, ranging among $g=\{22.5 ; 45 ; 67.5\}$ sec.

In this case, the traffic light cycle length has been considered to be $C_{\mathrm{p}}=90$ seconds. Since the time headway $(H=5 \mathrm{~min})$ is not multiple of the cycle time of traffic lights, buses arrive at intersections at different times of the red-green signal sequence. This fact may worsen the bus headway adherence, even when no exogenous disturbance is generated (base line scenario). The results are summarized in Figure 7 considering the departure of 27 consecutive buses from bus stop $s=1$. All instances with equal green time $(g)$ are presented together. The relative increment of all performance indicators $\left(T P T, Z_{O}, Z_{T}, c_{v}\right)$ are roughly equal in those instances with the same green time allocation. The spacing between intersections seldom affects the behavior of the control strategies for a given traffic light setting. The variations of all indicators are lower than $5 \%$, except for Strategy $S 1$, where these differences are up to $10 \%$.

Therefore, the differential behavior of control strategies is only identified for instances with different green time settings. When the green time is equal to $g=67.5 \mathrm{~s}\left(g / C_{p}=0.75\right)$, the bus delays at intersections generate by themselves low headway variations. In this situation (base line scenario), the level of service can be stated as $\operatorname{LoS} \mathrm{B}\left(c_{v}=0.28\right)$. The generation of a time disturbance of $U=4$ min to vehicle $j=3$ (Uncontrolled scenario) makes the system more unstable, increasing the variation of headways up to $c_{v}=0.72(\operatorname{LoS} \mathrm{D})$. Strategy $S 0$ significantly reduces travel time of users with regard to no control scenario, at expenses of increasing operating costs, deploying more vehicles.

Strategy $S 1$ with no slacks is not effective, since the total cost of the system (agency and users) is 1.15 times greater than the base line scenario. Vehicles often arrive at intersection when the green phase is active $\left(\mathrm{g} / C_{p}=0.75\right)$, so that the efficiency of strategy $\mathrm{S} 2$ is still limited. This strategy cannot improve the values of all performance indicators in the base line scenario. The best control criteria is strategy S2 with slack of $\phi_{s}=3 \mathrm{~min}$, characterized by $\mathrm{c}_{\mathrm{v}}=0.24$ and a total cost of 1.019 times greater than baseline scenario. It is remarkable that Strategy S0 with $\phi_{S}=3$ min presents similar results as the former one.

The analysis of the bus performance when $g=45 \mathrm{sec}\left(g / C_{p}=0.5\right)$ is essentially different. In the baseline case, the stoppings of vehicles at intersections significantly increase the bus bunching phenomena, presenting $c_{v}=0.75$. It can be stated as LoS F. Therefore, the creation of an exogenous service disruption $U=4$ min in the Uncontrolled scenario just worsens the total cost by $6.5 \%$ and seldom increases the bus bunching effect $\left(c_{v}=0.82\right)$. 


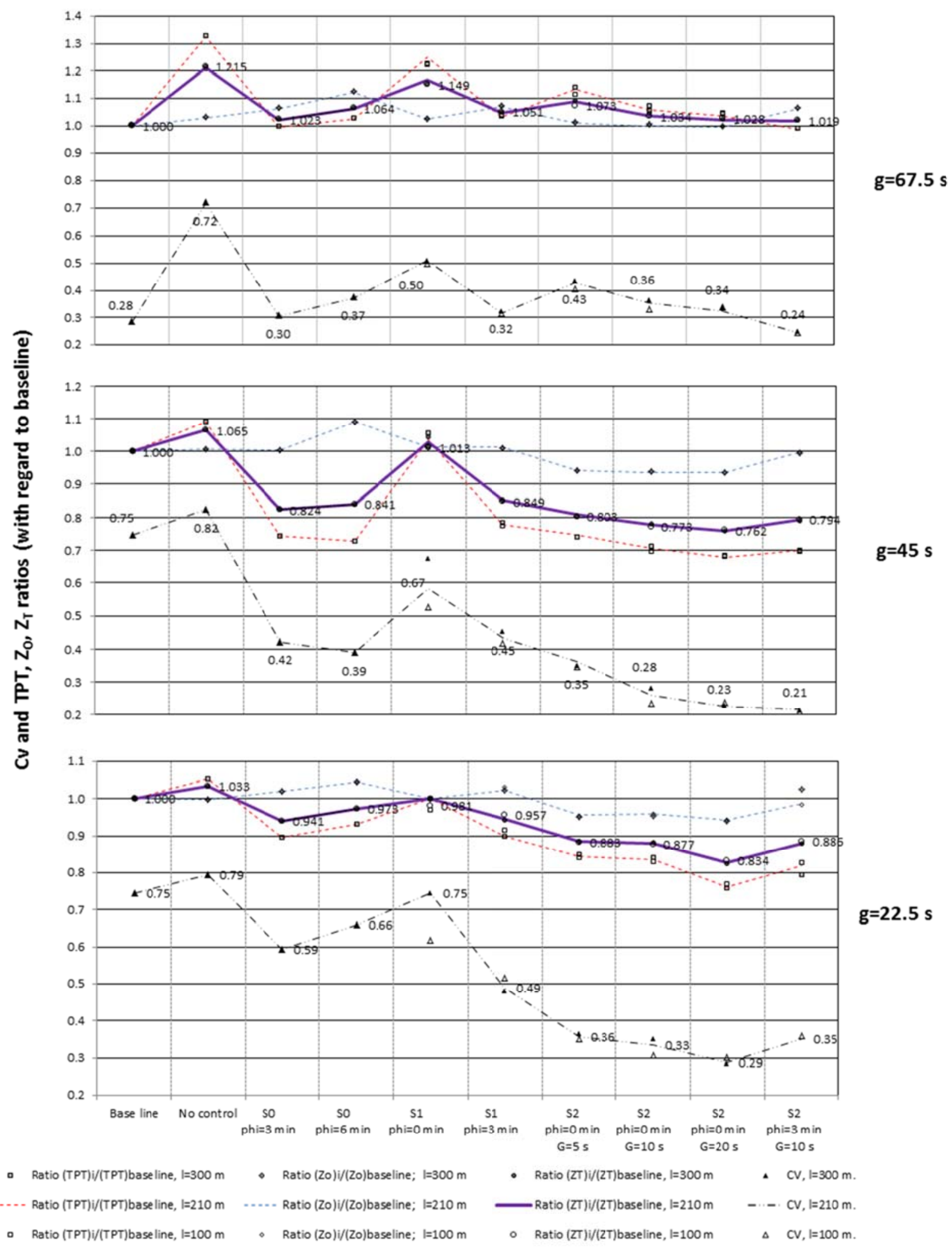

FIGURE 7. Sensitivity analysis of the performance of buses in Problem 1.3 when $g=22.5 ; 45$ and 67.5 sec.

In strategy $S 0$, a slack time of $\phi_{s}=3 \mathrm{~min}$ is sufficient to reduce the total cost by $17.6 \%$ (with regard to base line) and the variation of headways at $\mathrm{c}_{\mathrm{v}}=0.42$. Strategy $S 0$ with $\phi_{s}=6$ min reduces the travel time of users at the expenses of increasing the operating cost, so that this strategy is more expensive considering total cost. 
Strategy $S 1$ with no slacks is able to enhance the system performance with regard to the uncontrolled scenario, but the cost savings achieved are far away from those of strategy S0. Finally, strategy $S 2$ really outperforms the previous strategies. When the green extension length is $G=5 \mathrm{sec}$, this strategy is able to reduce both operating and user costs with regard to baseline scenario. As a result, the total cost is diminished by $20 \%$ and the service regularity can be graded as $\operatorname{LoS}=\mathrm{B}\left(c_{v}=0.35\right)$. If we continuously increase the green extension length, the performance of this strategy is outstanding, with total cost savings ranging from $20-34 \%$ and $\mathrm{c}_{\mathrm{v}}<0.39$ (LoS B or C). Nevertheless, the hybrid strategy $S 2$ with slacks $\left(\phi_{s}=3 \mathrm{~min}\right)$ presents the lowest coefficient of headway variation at the expenses of introducing one extra vehicle, increasing operating costs with regard to strategies without slack times.

Eventually, the results obtained when the green time at intersections is $g=22.5 \mathrm{sec}$ are fairly similar to those presented when $g=45 \mathrm{sec}$. Strategy S2 outperforms the indicators of other available strategies. However, the total cost savings are not as high as the former ones, ranging among $13-16 \%$ while the variation of headways are maintained between $c_{v}=0.30-0.36$. This strategy is able to reduce user cost, whereas maintaining the number of vehicles needed concerning the baseline scenario. Vehicles can be speeded up avoiding potential delays at intersections. This fact causes, in some instances, even lesser operating cost than baseline scenario.

\subsubsection{Unstable motion created by traffic light settings, traffic flows and demand rates at stops (Problem 3)}

If we consider the test instance representing the local bus route of highest demand in Barcelona (H6 route), the results generally follow the same pattern explained above. The simulation is carried out during the peak morning time (6.00-9.00 AM). In that case, there is no disturbance artificially-generated since real traffic light control, heterogeneous user arrival rates at stops and car traffic flow, tend to make the headway adherence unstable. In this case, we implement hybrid strategies based on the provision of slack time at headers together with the dynamic implementation of strategies $S 1$ or $S 2$. Figure 8 summarizes the simulated metrics in that route for different slack times at last stops ( $\phi_{s}=1,3$ and 6 minutes). The implementation of higher slack times generally improves both travel times and the headway adherence of the corridor, when only the static control strategy is considered (strategy $S 0$ ). However, the case with $\phi_{s}=6 \mathrm{~min}$ does not provide any benefits compared to the instance when $\phi_{s}=3 \mathrm{~min}$. It reflects that this strategy is neither scalable nor adaptable. Both travel times and headway adherence remain roughly constant for slack times greater than a minimum threshold. It is worth mentioning that the coefficients of headway variation when strategy S0 is implemented are greater than $c_{v}=0.75$, which corresponds to the Level of Service F (taking into account the classification of TRB, 2013). The introduction of dynamic controlling strategies significantly outperforms the performance of the bus network. Strategy $S 1$ reduces the total cost by $14-28 \%$ and $c_{v}$ by $27-58 \%$ with regard to the static controlling strategy with low slacks (strategy $S 0 \phi_{s}=1 \mathrm{~min}$ ). The level of service in that situation with regard to the headway adherence criterion is $\mathrm{E}\left(\phi_{s}=1 \mathrm{~min}\right)$ and $\mathrm{D}\left(\phi_{s}=3\right.$ or $\left.6 \mathrm{~min}\right)$. However, when traffic light priority is activated for buses (strategy $S 2$ ), the results are outstanding and outperform those provided by the speed modification controlling strategy (strategy $S 1$ ). Strategy $S 2$ improves $T P T, Z_{T}$ and coefficient of headway variation by $40-41 \%, 38-39 \%$, $72-80 \%$ respectively, with regard to the strategy $S O$. The time adherence variable can be controlled with strategy $S 2$ below the threshold $c_{v}<0.3$, which corresponds to level of service A or B. The regularity effects of dynamic controlling strategies on the bus service can be observed in Figure 9 a-c. Bus trajectories in the route direction A-B (Zona Universitària-Fabra $\mathrm{i}$ Puig) are depicted when the slack time at the ending stop is $\phi_{s}=1 \mathrm{~min}$. Although the inclusion of higher slack times $\left(\phi_{s} \geq 6 \mathrm{~min}\right.$ ) would improve the results of strategy $\mathrm{S} 1$, the actual performance of the service with strategy $S 2$ does not get better with those slack times. Therefore, full dynamic bus controlling strategy (strategy $S 2$ ) does not need any unproductive slack time at holding points in order to guarantee good headway adherence and user travel times. 


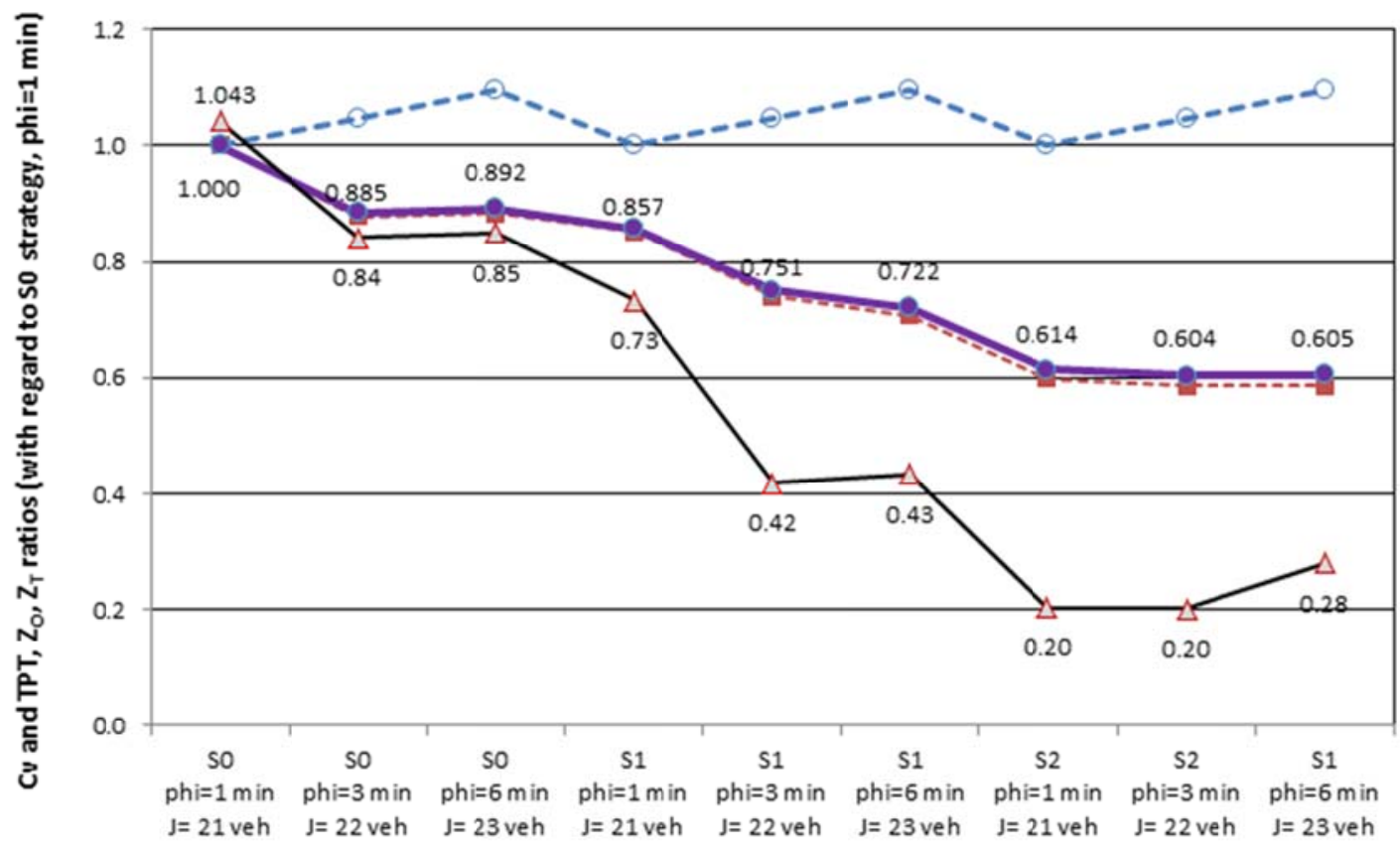

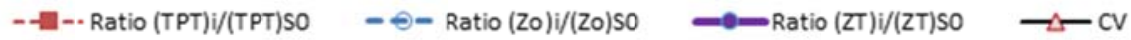

FIGURE 8. Total user travel time and headway adherence in bus route $\mathrm{H6}$ considering different slack times at ending stops
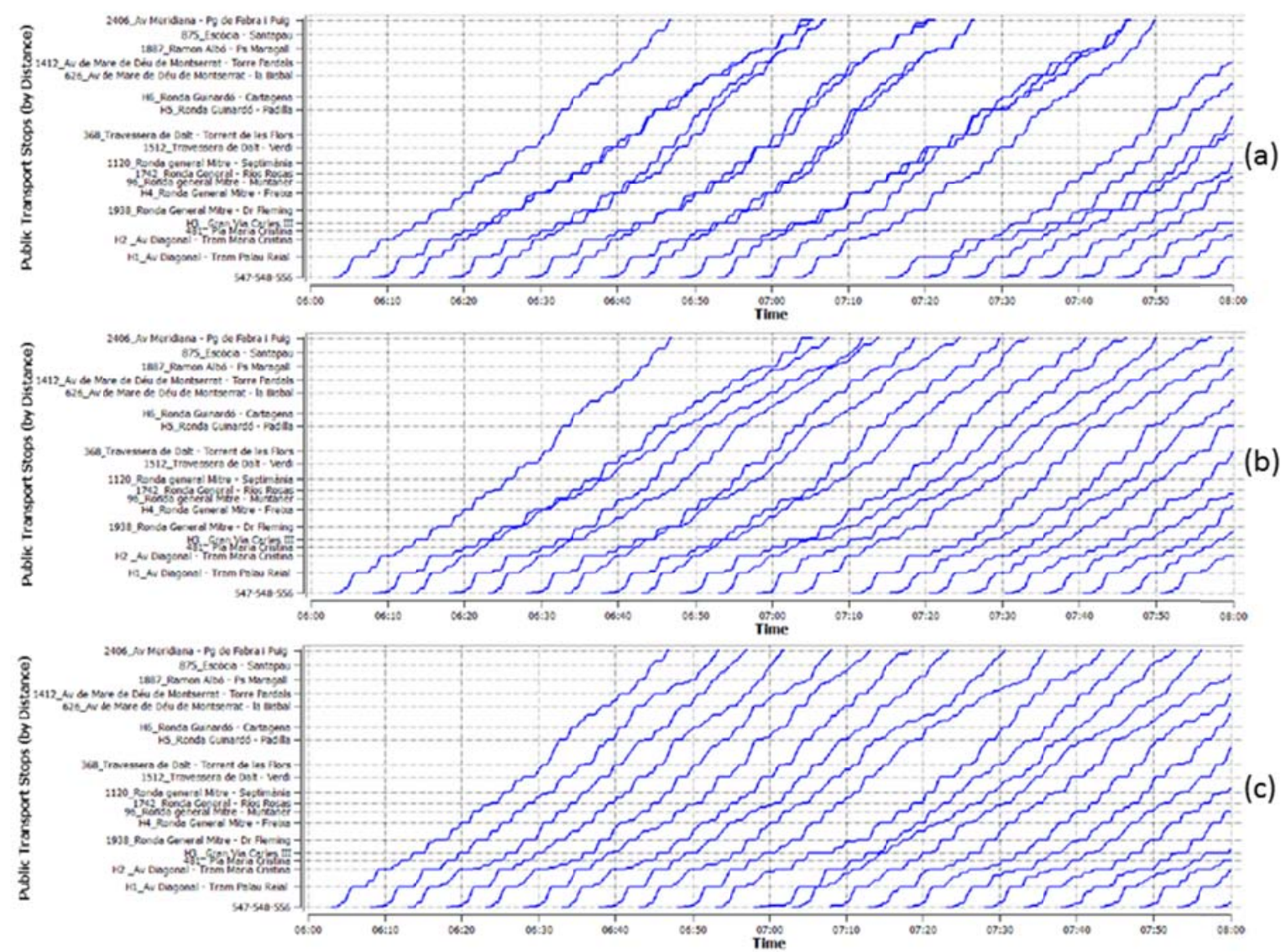

FIGURE 9. Simulation of bus trajectories in route H6 (direction Zona Universitària-Fabra i Puig) when $\phi \mathrm{s}=1$ min. a) Strategy S0, b) Strategy S1, c) Strategy $S 2$. 


\section{CONCLUSIONS}

In this paper we analyzed the efficiency of control strategies of bus bunching based on the user performance and operating costs incurred by the transit agency to run the service. An operational model was presented to reproduce how time disruptions propagate along the bus route when no controlling strategy is implemented. The model advances the contributions of Daganzo (2009) since it takes into account the vehicle capacity constraint in the formulations. Moreover, the modeling formulations could also estimate the deployment of existing controlling strategies to compensate disruptions: $S O$ introduced slack times at holding points (bus headers) and $S 1$ modified the cruising speed of buses at each stop to maintain the targeted headway in a similar way presented in Daganzo (2009). Since strategy $S 1$ actively reduced the speed of buses (i.e. it is impossible that buses run above the maximal cruising speed $v$ ), several buses would experience a higher round trip cycle time than the theoretical one. To overcome this problem, we proposed a new strategy $(S 2)$ that allowed only delayed buses (i.e. those with a higher time-headway with the vehicle ahead than the target value $H$ ) to be benefited by traffic light priority so that they could speed up.

The results showed that the propagation of even a small disturbance in a specific bus produces irregular vehicle arrivals at stops, causing extra operating costs, user costs and coefficient of headway variation increment. Indeed, when the vehicle departures from terminals are not synchronized with traffic lights, the system does not need any exogenous to present an unstable performance. In these situations, the time headway adherence worsens as the percentage of green phase at intersections is reduced. The coefficients of headway variation may rise to $c_{v} \approx 0.75$ when the percentage of green time at intersections is $g / C_{p} \leq 0.45$. Therefore, transit managers should define a target time-headway compatible with the light cycle times in the bus corridor to reduce bus bunching.

Vehicle capacity is an endogenous attribute of the system that contributes to mitigate the propagation of delays and the unstable motion of buses without exogenous controlling measures. When the delayed vehicle has enough capacity to accommodate the overall waiting passengers, the system tends to be more unstable. Any disturbance produces a dramatically increase of passenger travel times. However, the effects in operating costs are softened. On the other hand, if we consider vehicle capacity constraint, the total cost increases by almost $50 \%$ with regard to the idealized performance with perfect regularity.

Strategy $S 2$ resulted to be the best control method in terms of total passenger travel time, operating costs and total costs. This fact justifies the need of speeding up delayed buses when adaptive cruising speed modification is performed. The effectiveness of this strategy does not depend on the number of intersections but on the traffic light settings: the green time $(g)$ and the green extension $(G)$. The total cost savings of this control strategy are essentially more relevant when vehicle departures are not synchronized with signal settings and for corridors with low percentage of green time at intersections (i.e. the most unstable motion of buses considered). The coefficient of headway variation can be slightly higher to the minimal one obtained with strategy $S 0$ with larger slacks, unless headway variations range among level of service A or B. Nevertheless, Strategy $S 2$ only provided competitive results when the green extension time was significant $(G \geq 10 \mathrm{sec})$. This fact would produce negative effects on the traffic and passenger flow in the streets near to the intersections. Therefore, when the bus route under analysis runs along a corridor with important traffic volumes in the crossing streets, we recommend hybrid control strategies. They consist of providing minimal slack times (less than 2-3 minutes) in the bus schedule as well as implementing dynamic strategy $S 2$ with a green extension time of $G=10$ seconds, to recover larger and unpredictable disruptions. Hybrid strategies were the second best control alternative, only outperformed by Strategy $S 2$ with $G=20 \mathrm{sec}$. They generally increase the total cost of the system by at most $4 \%$ in problems with vehicle capacity constraint, with regard to Strategy $S 2$ with no slacks.

The control strategy $S O$ based on holding points is effective to maintain the bus performance at the same level of service as in the baseline case in ideal problems. However, it requires much slack time to control the system performance (6 minutes at terminals) for routes with high disruptions as well as high passenger flow. For medium-demand problems, lower slacks ( 3 minutes) seem to be sufficient to guarantee a similar total passenger travel time when no disturbance takes place. Therefore, it is a myopic, not-adaptive strategy since bus managers can not define in advance a minimal slack time to tackle the potential deviations that can appear. This strategy can keep total travel times stable at the expense of increasing operating cost.

The effectiveness of the control strategy $S 1$ is significantly dependent on the stabilization parameters $f_{f}$ and $f_{r}$ that reduce the cruising speed proportional to the headway deviation. Although this strategy keeps the number of resources constant in comparison to strategy $S O$, it was unable to alleviate the effects of bunching on the total travel time of users. Consequently, in capacitated problems, this strategy presents higher total costs than strategies with slack time. Strategy $S 1$ only outperformed the results provided by Strategy $S O$ with low slack 
times when the vehicle capacity was supposed to be unlimited. This situation corresponds to the idealistic hypothesis considered in Daganzo (2009), where this cruising speed modification strategy was presented.

Throughout the paper we assumed that: i) a delayed bus could not be overtaken by other buses and ii) the boarding time per passenger was considered to be constant and independent to the vehicle occupancy and the number of passengers waiting at stops. If assumption ii) is considered true and if we allow the model to consider overtaking, the results will not differ from the presented in the paper. However, if assumption ii) is substituted by variable unit boarding times, considering the crowdedness of stops and vehicles, the overtaking of buses may produce better results in passenger travel and waiting times. Moreover, another important assumption that deserves mentioning is that the passenger arrival rate at each stop was deterministic and constant during a predetermined stationary period of time. Therefore, the source of instability analyzed in this paper was any exogenous disruption that incremented the running time between two consecutive stops and the delays at signaled intersections. Here, the model can be further improved if stochastic passenger arrivals are considered, as presented in Bowman and Turnquist (1981) or Fonzone et al. (2015).

\section{ACKNOWLEDGEMENTS}

This paper is partially supported by the Spanish Economic and Competitiveness Ministry project, ENTROPIA TRA 2012-39466-C02-01.

\section{REFERENCES}

Adamski, A., Turnau, 1998. A. Simulation support tool for real-time dispatching control in public transport. Transportation Research Part A, vol. 32 (2), pp.73-87.

Argote-Cabanero, J., Daganzo, C.F., Lynn J.W. 2015. Dynamic control of complex transit systems. Transportation Research Part B: Methodological, Vol. 81, Part 1, 146-160.

Barnett, A., 1974. On controlling randomness in transit operations. Transportation Science 8 (2), $102-116$. Bartholdi III, J. J., Eisenstein, D. D. 2012. A self-coordinating bus route to resist bus bunching. Transportation Research Part B: Methodological, Vol. 46, Issue 4, 481-491.

Cortés, C.E., Sáez, D., Milla, F., Riquelme, M., Núñez, A., 2010. Hybrid predictive control for real-time optimization of public transport system' operations based on evolutionary multiobjective optimization.

Transportation Research Part C-Emerging Technologies 18 (5), 757-769.

Daganzo, C.F., 2009. A headway-based approach to eliminate bus bunching: Systematic analysis and comparisons. Transportation Research Part B 43 (10), 913-921.

Daganzo, C.F., Pilachowski, J., 2011. Reducing bunching with bus-to-bus cooperation. Transportation Research Part B 45 (1), 267-277.

Delgado, F., Muñoz, J.C., Giesen, R., 2012. How much can holding and/or limiting boarding improve transit performance? Transportation Research Part B 46 (9), 1202-1217.

Dessouky, M., Hall, R., Zhang, L., Singh, A., 2003. Real-time control of buses for schedule coordination at a terminal. Transportation Research Part A, vol. 37, 145-164.

Eberlein, X.J., Wilson, N.H.M., Bernstein, D., 2001. The holding problem with real-time information available. Transportation Science 35 (1), 1-18.

Fonzone, A., Schmöcker, J. D., Ronghui, L., 2015. A model of bus bunching under reliability-based passenger arrival patterns. Transportation Research Part C, http://dx.doi.org/10.1016/i.trc.2015.05.020.

Hill, S.A., 2003. Numerical analysis of a time -headway bus route model. Physica A: Statistical Mechanics and its applications, vol. 328 (1-2), pp. 261-273.

Hu, J., Park, B.B., Lee, Y., 2015. Coordinated transit signal priority supporting transit progression under Connected Vehicle Technology. Transportation Research Part C 55 393-408.

Muñoz, J.C., Cortés, C.E., Giesen, R., Sáez, D., Delgado, F., Valencia, F., Cipriano, A., 2013. Comparison of dynamic control strategies for transit operations. Transportation Research Part C 28, 101-113.

Newell, G.F., Potts, R.B., 1964. Maintaining a bus schedule. In: Proceedings of the 2nd Australian Road Research Board, vol. 2, pp. 388-393.

Osuna, E. E., Newell, G. F., 1972. Control strategies for an idealized public transportation system.

Transportation Science 6, 52-72.

TRB (Ed.), 2013. Transit Capacity and Quality of Service Manual. 3rd Edition. Transit Cooperative Research Program, Transportation Research Board, Washington, DC.

Turnquist, M.A., 1981. Strategies for improving reliability of bus transit service. Transportation Research Record 818, pp. 25-29. 
Rossetti, M.D., Turitto, T., 1998. Comparing static and dynamic threshold based control strategies. Transportation Research Part A, vol. 32 (8), pp.607-620.

Sáez, D., Cortés, C., Riquelme, M., Núñez, A., Milla, F., Tirachini, A., 2012. Hybrid predictive control strategy for a public transport system with uncertain demand. Transportmetrica 8 (1), 61-86.

Xuan, Y., Argote, J., \& Daganzo, C.F. (2011). Dynamic bus holding strategies for schedule reliability: Optimal linear control and performance analysis. Transportation Research Part B: Methodological, Vol. 45, (10), 18311845.

Yu, B., Yang, Z., 2007. A dynamic holding strategy in public transit systems with real-time information. Applied Intelligence 31 (1), 69-80. 
Appendix. Traffic light settings of Problem 3.

\begin{tabular}{|c|c|c|c|c|}
\hline \multicolumn{5}{|c|}{ Direction: Fabra i Puig to Zona Universitaria } \\
\hline Intersection name & $\begin{array}{r}\begin{array}{r}\text { Signal } \\
\text { phase }\end{array} \\
\end{array}$ & $\begin{array}{r}\text { Green Time } \\
(g, \text { in seconds })\end{array}$ & $\begin{array}{r}\text { Cycle }\left(C_{p},\right. \\
\text { in seconds }) \\
\end{array}$ & $\begin{array}{r}\text { Green time } \\
\text { percentage }\left(g / C_{p}\right)\end{array}$ \\
\hline Meridiana - Fabra I Puig & 4 & 3 & 100 & $3 \%$ \\
\hline Fabra i Puig - Arnau d'Oms & 5 & 37 & 91 & $41 \%$ \\
\hline Fabra i Puig - Pi i Molist & 5 & 17 & 91 & $19 \%$ \\
\hline Av. Borbó - Costa i Cuxart & 7 & 34 & 90 & $38 \%$ \\
\hline Av. Borbó - Pg. Maragall - Av. Mare de Déu Montse & 6 & 28 & 91 & $31 \%$ \\
\hline Rda. Guinardó - Cartagena & 5 & 15 & 91 & $16 \%$ \\
\hline Rda. Guinardó - Padill - Túnels & 5 & 16 & 108 & $15 \%$ \\
\hline Rda Guinardó - Tunels Rovira - Lepant & 5 & 25 & 91 & $27 \%$ \\
\hline Rda. Guinardó - Pi i Maragall - Pl. Alfons el Savi & 5 & 44 & 91 & $48 \%$ \\
\hline Trav. Dalt - Escorial & 5 & 66 & 120 & $55 \%$ \\
\hline Trav. Dalt - Massens - St. josep muntanya & 5 & 75 & 120 & $63 \%$ \\
\hline Trav. Dalt - Verdi & 5 & 84 & 120 & $70 \%$ \\
\hline Trav. Dalt - Torrent de l'Olla & 5 & 84 & 120 & $70 \%$ \\
\hline Mitre - Vallirana - Padua & 5 & 63 & 108 & $58 \%$ \\
\hline Mitre - Balmes & 5 & 41 & 108 & $38 \%$ \\
\hline Mitre -Muntaner & 5 & 35 & 108 & $32 \%$ \\
\hline Mitre - Mandri & 5 & 39 & 108 & $36 \%$ \\
\hline Mitre - Ganduxer - Freixa & 5 & 55 & 108 & $51 \%$ \\
\hline Mitre - Augusta & 5 & 50 & 108 & $46 \%$ \\
\hline Mitre - Fleming & 5 & 50 & 108 & $46 \%$ \\
\hline Mitre -Dr Roux & 5 & 60 & 108 & $56 \%$ \\
\hline Pl. Prat de la Riba & 5 & 14 & 108 & $13 \%$ \\
\hline Pg. Manuel Girona - Capita Arenas & 7 & 37 & 85 & $44 \%$ \\
\hline Diagonal / Maria Cristina & 1 & 26 & 120 & $22 \%$ \\
\hline Puis XII est & 1 & 34 & 120 & $28 \%$ \\
\hline Pius XII oest & 1 & 34 & 120 & $28 \%$ \\
\hline Diagonal - Gregorio Marañon & 1 & 78 & 150 & $52 \%$ \\
\hline
\end{tabular}




\begin{tabular}{|l|r|r|r|r|}
\hline \multicolumn{5}{|c|}{ Direction: Zona Universitaria to Fabra i Puig } \\
\hline Intersection name & $\begin{array}{r}\text { Signal } \\
\text { phase }\end{array}$ & $\begin{array}{r}\text { Green Time }(g, \\
\text { in seconds })\end{array}$ & $\begin{array}{r}\text { Cycle }\left(C_{p},\right. \\
\text { in seconds })\end{array}$ & $\begin{array}{r}\text { Green time } \\
\text { percentage }\left(g / C_{p}\right)\end{array}$ \\
\hline Gonzalez Tables - Diagonal & 1 & 29 & 150 & $19 \%$ \\
\hline Diagonal - Gregorio Marañon & 1 & 78 & 150 & $52 \%$ \\
\hline Pius XII oest & 1 & 34 & 120 & $28 \%$ \\
\hline Puis XII est & 1 & 34 & 120 & $28 \%$ \\
\hline Diagonal / Maria Cristina & 1 & 34 & 120 & $28 \%$ \\
\hline Mitre -Dr Roux & 5 & 60 & 108 & $56 \%$ \\
\hline Mitre - Fleming & 5 & 50 & 108 & $46 \%$ \\
\hline Mitre - Augusta & 5 & 50 & 108 & $46 \%$ \\
\hline Mitre - Ganduxer - Freixa & 5 & 55 & 108 & $51 \%$ \\
\hline Mitre - Mandri & 5 & 39 & 108 & $36 \%$ \\
\hline Mitre -Muntaner & 5 & 35 & 108 & $32 \%$ \\
\hline Mitre - Balmes & 5 & 41 & 108 & $38 \%$ \\
\hline Mitre - Vallirana - Padua & 5 & 63 & 108 & $58 \%$ \\
\hline Trav. Dalt - Torrent de l'Olla & 5 & 84 & 120 & $70 \%$ \\
\hline Trav. Dalt - Verdi & 5 & 84 & 120 & $70 \%$ \\
\hline Trav. Dalt - Massens - St. josep muntanya & 5 & 75 & 120 & $63 \%$ \\
\hline Trav. Dalt - Escorial & 5 & 66 & 120 & $55 \%$ \\
\hline Rda. Guinardó - Pi i Maragall - Pl. Alfons el Savi & 5 & 44 & 91 & $48 \%$ \\
\hline Rda Guinardó - Tunels Rovira - Lepant & 5 & 25 & 91 & $27 \%$ \\
\hline Rda. Guinardó - Padill - Túnels & 5 & 16 & 108 & $15 \%$ \\
\hline Rda. Guinardó - Cartagena & 5 & 15 & 91 & $16 \%$ \\
\hline Maragall - Ramon Albó & 5 & 10 & 91 & $11 \%$ \\
\hline Felip II - Ramon Albó & 5 & 41 & 91 & $45 \%$ \\
\hline Arnau d'Oms - Escòcia & 6 & 45 & 91 & $49 \%$ \\
\hline Meridiana - Escocia - Dublin & 36 & 110 & $33 \%$ \\
\hline
\end{tabular}

